جامعة مدينة السادات كلية الحقوق الار اسات العليا قسم القانون الخاص ماهية حقوق ارتفاق الطاقة الثمسية (دراسة مقارنة)

في ضوء القوانين الأمريكية والمصرية بحث مقدم ضمن متطلبات الحصول علي درجة الدكتوراه في القانون

$$
\text { الباحث }
$$

\title{
حسن حواش حسن حواش
}

مدرس مساعد بقسم القانون الخاص (تخصص القانون المدني) كلية الحقوق - جامعة مدينة السادات $r .19$ 


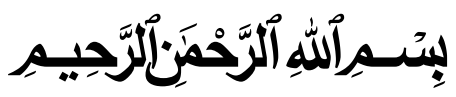

\section{صدق الله العظيم}

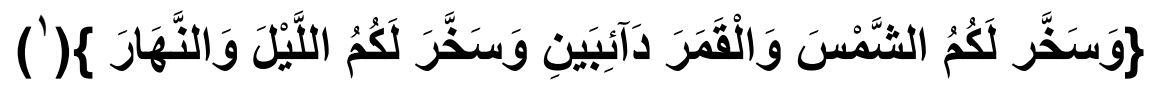

\section{المقدمة}

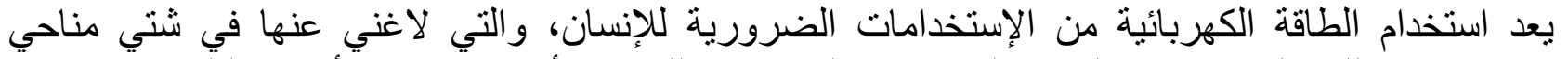

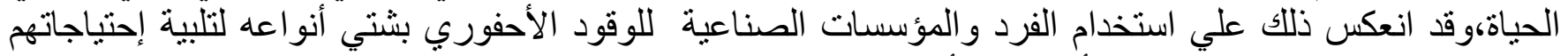

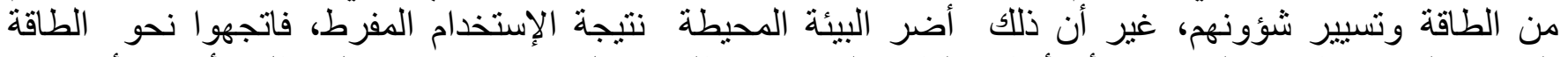

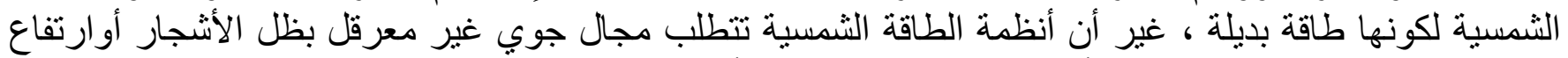

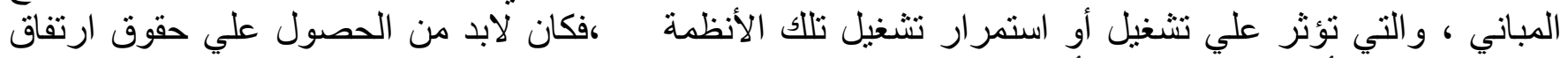

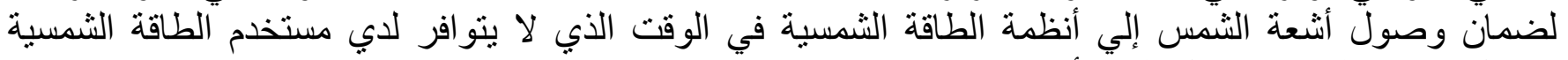
المجال الجوي الكافي لتشغيل تلك الأنظمة النظة

\section{أهمية موضوع الدراسة :}

تعتبر حقوق ارتفاق الطاقة الثمسية من المفاهيم المستحدثة ، و التي كانت نتاج التطور العلمي و التقني علي

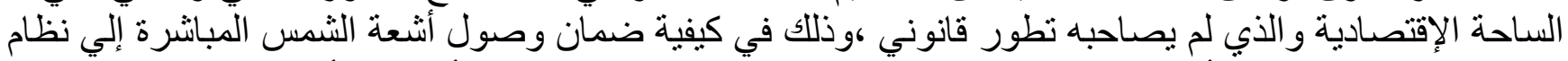

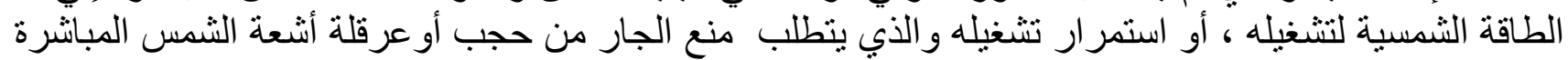

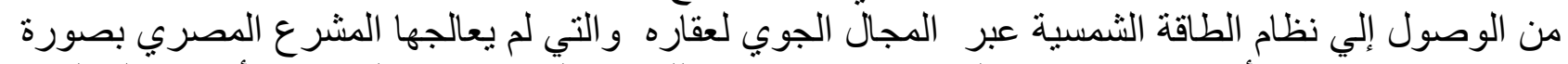

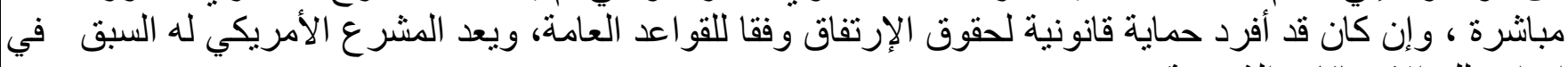
إبراز تلك الإرتفاقات الثمسية

\section{مشكلة الدر اسة : م م}

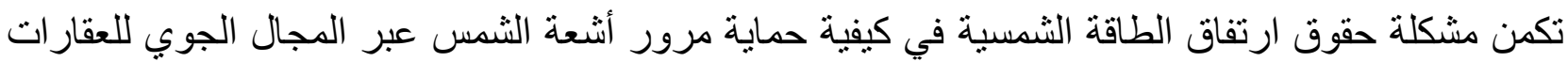

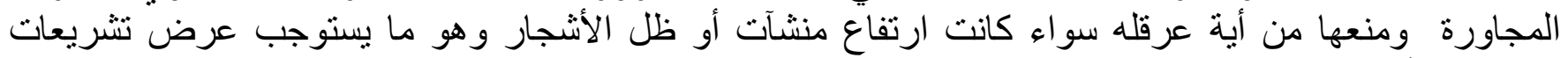
الو لايات الأمريكية التي نظمت تلألك الحقوق

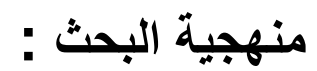

سنعتمد في تلاك الدراسة علي عدة مناهج بالنظر إلي الأبعاد التي تثير ها حقوق ارتفاق الطاقة الثمسية المنهج

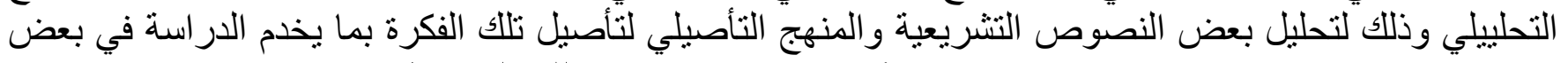

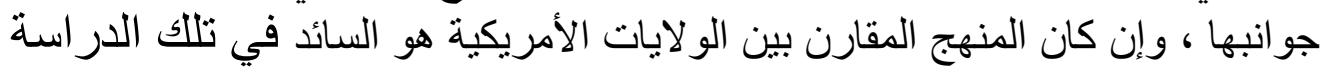


خطة الدراسة:نقسم الدراسة إلي مطلبين وذللك علي النحو التالي : المطلب الأول : مفهوم حقوق ارتفاق الطاقة الثمسية الفرع الأول : تعريف حقوق الإرتفاق في التشريعات المقارنة الفرع الثاني : تعريف حقوق ارتفاق الطاقة الثمسية : نمريف الفرع الثالث: عناصر حقوق ارتفاق الطاقة الثمسية الفرع الرابع:خصائص حقوق ارتفاق الطاقة الثمسية

المطلب الثاني : الأساس القانوني لحقوق ارتفاق الطاقة الثمسية الفرع الأول :التكييف القانوني لانظمة الطاقة الثمسية الفرع الثاني : الطبيعة القانونية لأثعة الثمس الفرع الثالث : التكييف القانوني لحقوق ارتفاق الطاقة الثمسية 


\section{المطلب الأول :مفهوم حقوق ارتفاق الطاقة الثمسيية}

\section{تمهي⿻ وتقسيم}

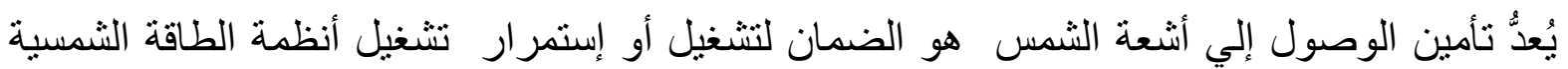

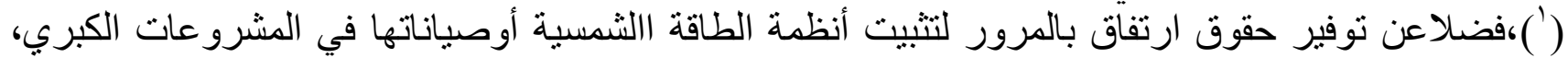

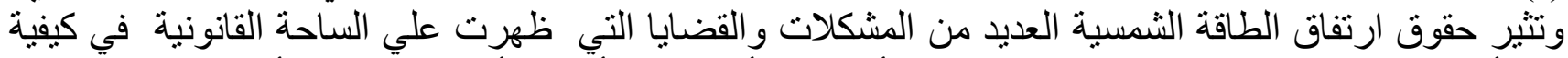

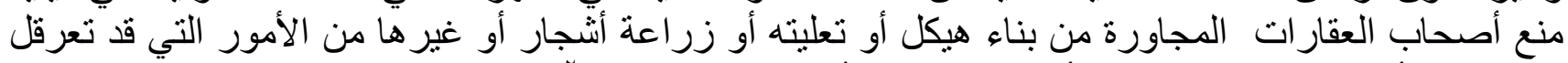

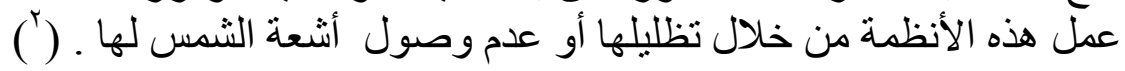

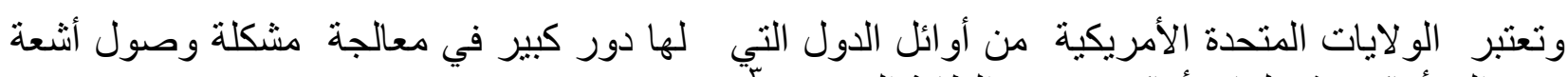

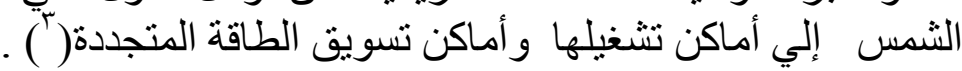

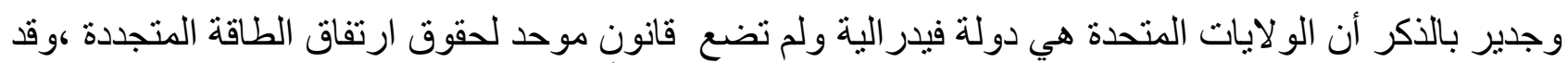

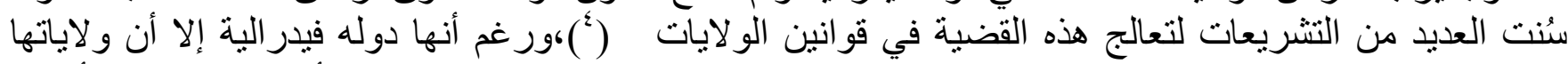

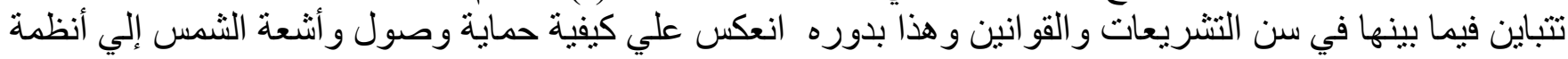

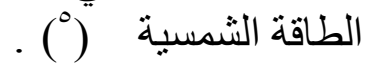

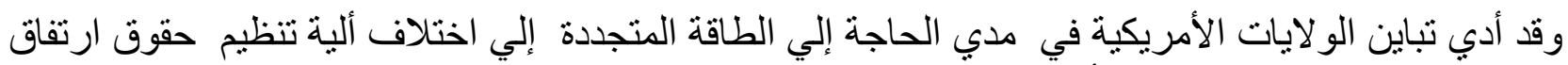

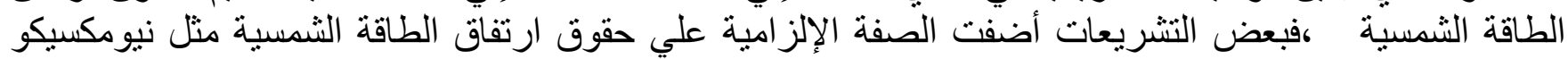

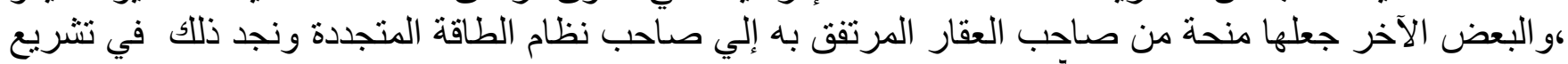
(') ولاية داكوتا الجنوبية وفرجينيا

وسوف نستعرض في هذا المطلب تعريف حقوق الإرتفاق وفقاً للتشريعات المقارنة يليه تعريف حقوق ارتفاق الطاقة الثمسية ثم يعقب ذللك عناصر ها وخصائصها كل في فرع الإنقاف مستقل علي النحو التالي :

(1)- See: James M. Pedowitz: SOLAR ENERGY EASEMENTS, Real Property, Probate and Trust (Journal, Vol. 15, No. 4,;1980,p:797

(2)-See: Endre Rex/ Some Financial And Legal Aspects Of Solar Energy Usage:-

http://pledgingforchange.com/green/some-financial-and-legal-aspects-of-solar-energy-usage.php( the last visi 15/7/2019

(3 )- James M. Pedowitz: Ibid:797

(4)-K.K DuViver "SOLAR SKYSpace B,UniferSity of Denver Sturm ,Collage of Law,2014.p:891 هـ ـ لمزيد من التفاصيل عن التشريعات التي تبنتّ حقوق ارتفاق الطاقة الثمسية ، راجع : Adrian J. Bradbrook: "Australian and American Perspectives on the Protection of Solar and Wind Access" Natural Resources Journal,VOL: 28,1988,P:237 -244

(6)- THE 2011Wisconsin . 386-2011 .Chapter : 700. 


\section{الفرع الأول : تعريف حقوق الإرتفاق في التشريعات المقارنة}

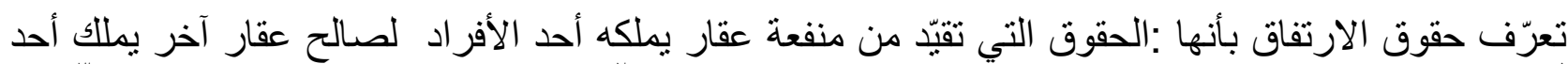

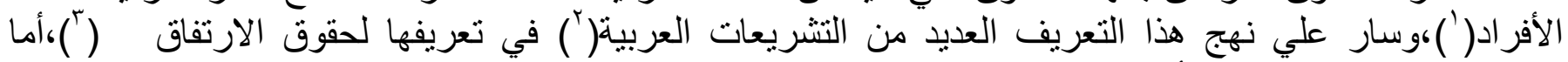
تشريعات بعض الولايات الأمريكية فقد تباينت في تعريفها لحقوق الارتفاق، فقدعرّف تشرئ تشريع ولاية داكوتا الجات الجنوبية

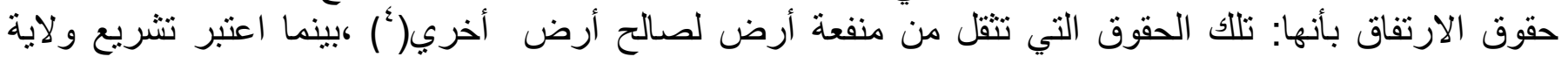

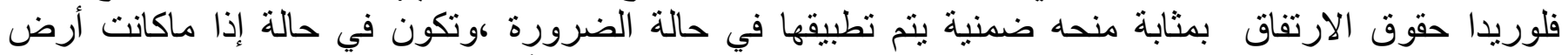

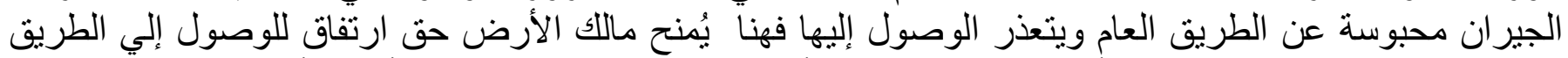

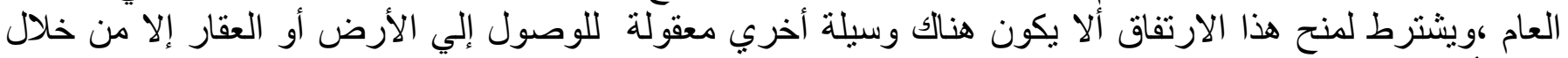
هذه الأرض الوض

وينص تشريع ولاية أوريجون علي أن حق الإرتفاق هي مصلحة تثقل عقار بفائدة لصالح عقار آخر مثل حق

(المرور ()

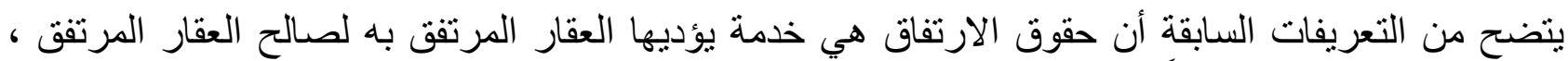

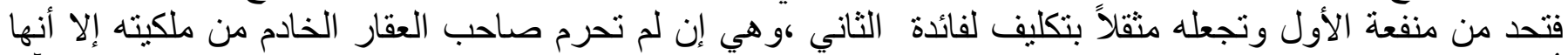

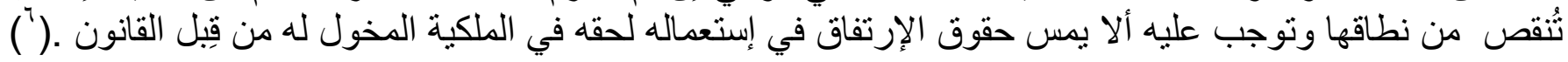

\section{الفرع الثاني : تعريف حقوق ارتفاق الطاقة الشمسية}

\section{(1) See:}

Donald R. Richards Knud E. Hermansen:" MAINE ROADS AND EASEMENTS" Maine Law Review journal, Volume: 48 , Number: 2 Article: 3, ,(2018),p:200" an easement as a right of use over the property of another. An easement is a right in the owner of one parcel of land, by reason of such ownership, to use the land of another for a special purpose not inconsistent with a general property right in the owner"

(') -ر اجع: محم وحيد الدين سوار - شرح القانون المدني ـ الحقوق العينية الأصلية ـ أسباب كسب الملكية والحقوق العينية

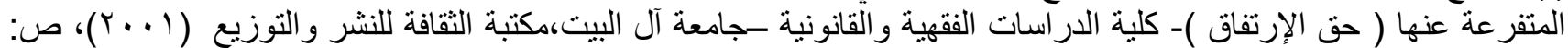

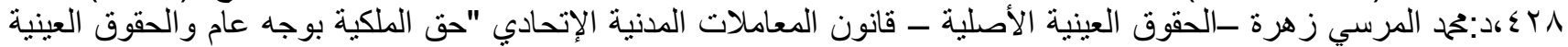

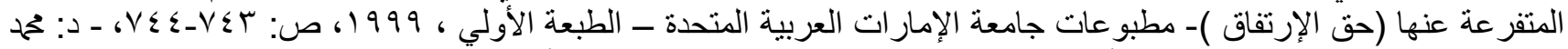

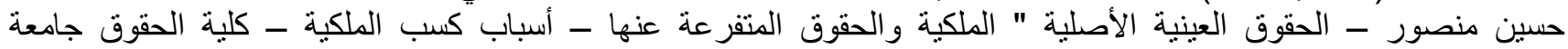

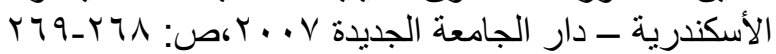

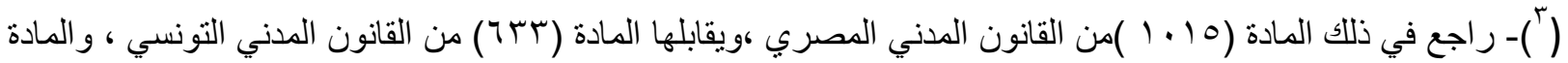

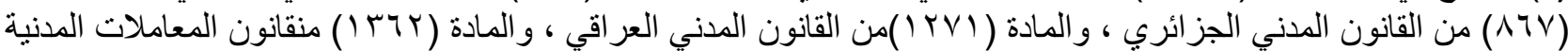

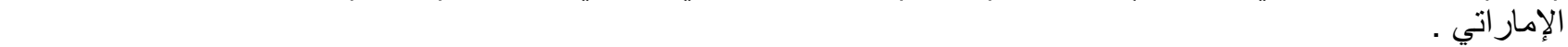
(4)- The 2018 South Dakota Codified Laws Title 43 - PROPERTY Chapter 13 - Easements And (Servitudes § 43-13-2 Easements-Definition.

(5) - The 2015 Oregon Revised Statutes Volume : 03 - Landlord-Tenant, Domestic Relations, Probate Chapter 105 - Property Rights Section 105.170 - Definitions(updates2017)

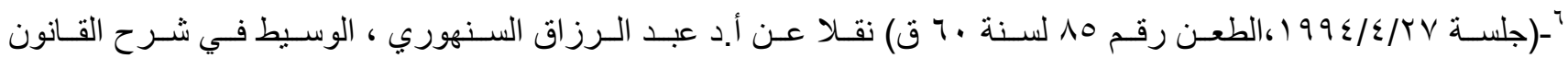

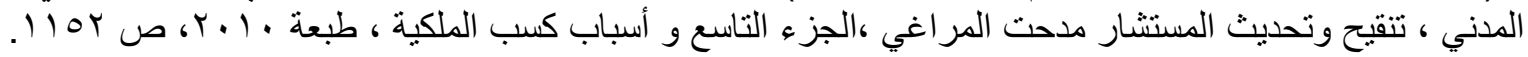




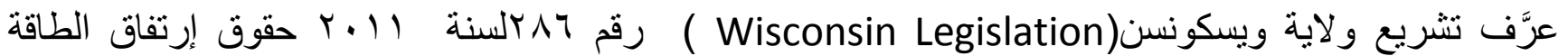

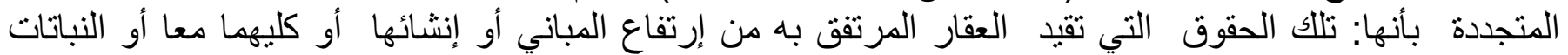

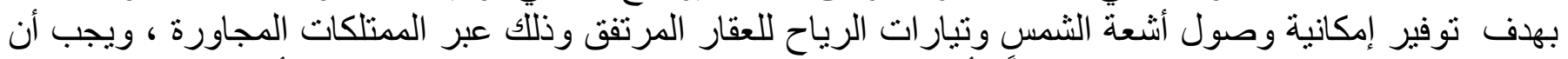

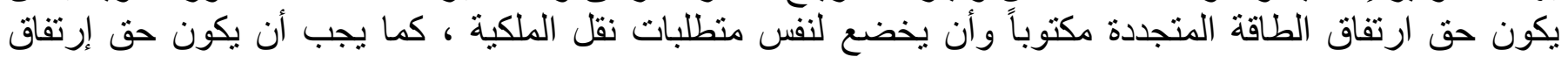

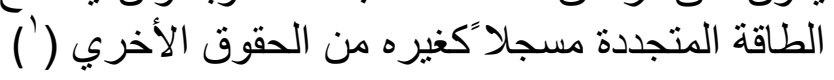

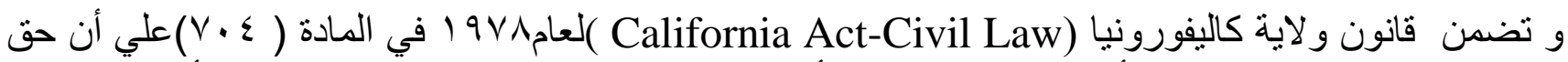

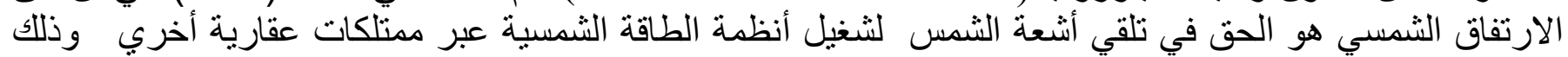

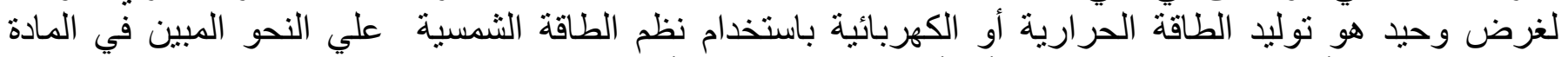

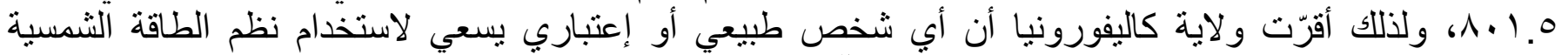

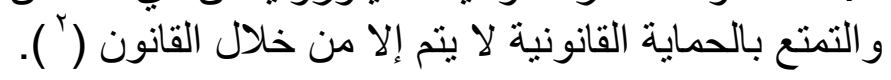

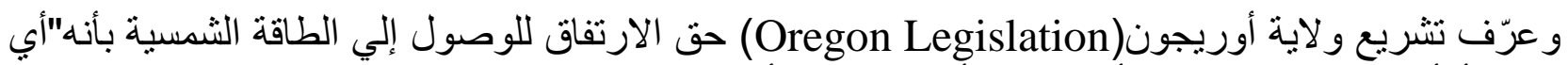

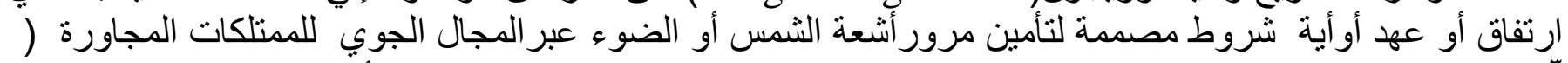

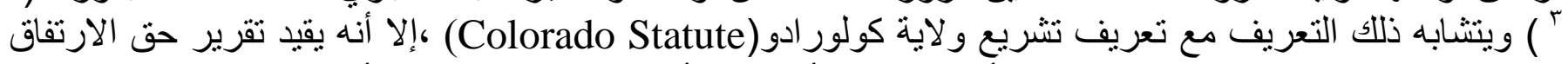

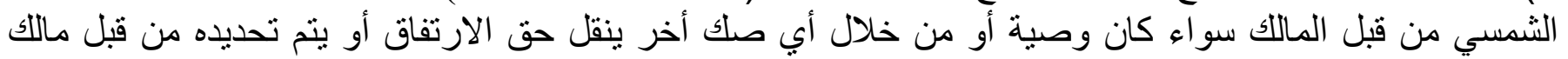

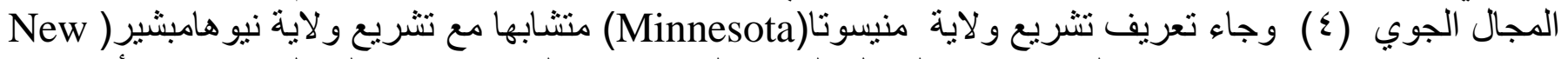

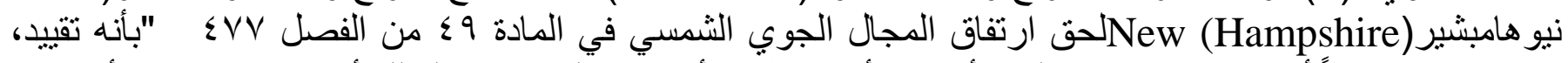

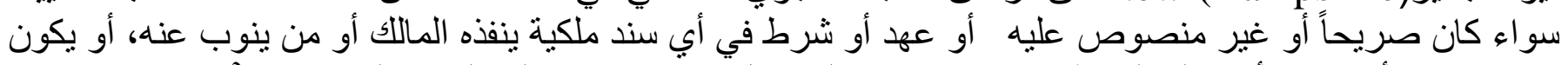

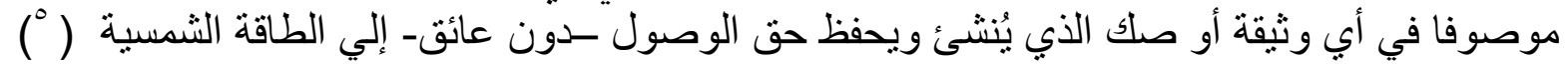

ثانيا :التعريف الفقهي:

عرّف جانب من الفقه الأمريكي حقوق ارتفاق الطاقة الثمسية بأنها : تللك القيود الاتفاقية أو القانونية التي تحمي التئي

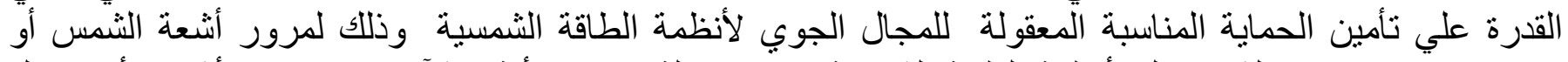
استمرار مرور ها من الثمس إلي أنظمة الطاقة الثمسية دون عرقلة من من أية منشآت سواء كانت أنشجار أو منازل

(1) $)^{-}$The 2011-386- Wisconosin chapter 700,Section 35(updates 2018). 700.35 Renewable energy resource easements. . In this section, "renewable energy resource easement" means an easements which limits the height or location ,or both ,of permissible development on the burdened land in terms of a structure or vegetation or both for the purpose of providing access for the benefited land to wind or sunlight passing over the burdened land .Every renewable energy resource easement shall be in writing and shall be subject to the same conveyancing and instrument recording requirements as other easements. Renewable energy resource easements shall run with the land benefited and burdened unless otherwise expressly stated therein

((2)- The 2018 California Act(civil code section 714

(3)- See: Eisenstadt ,Melvin M. "Access to Solar Energy :The Problem and its Current Status, Natural Resources JournalVol:22,1982,p.24

(4)- The 2017Colorado Rev.stst\&38-32-5-100.3(1)Solar easement "means the right of receiving sunlight across real property for any solar energy device. such a right may be stated in any deed ,will ,or other instrument executed by or on behalf of any owner of land or sky space

(5)- the 1978Califronia Act-Civil Law(updates 2018) 


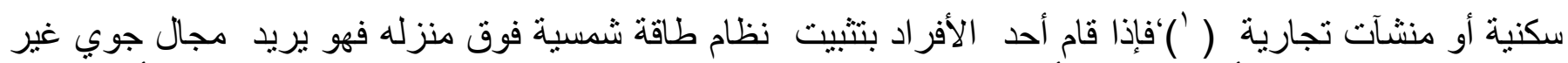

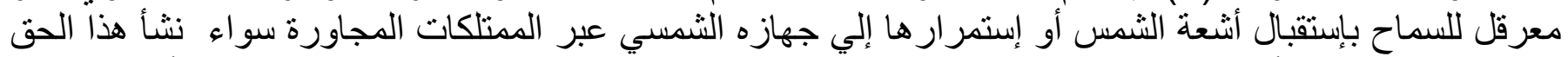
من خلال اتفاق بين أصحاب حق الارتفاق (صاحب نظام الطاقة الثمسية وصاحب العقار المجار المجاور ) أو من خلال

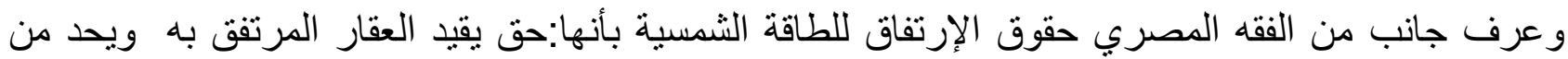

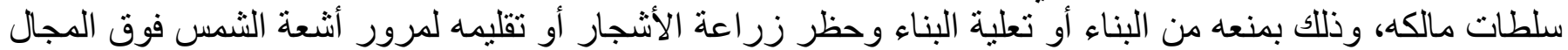

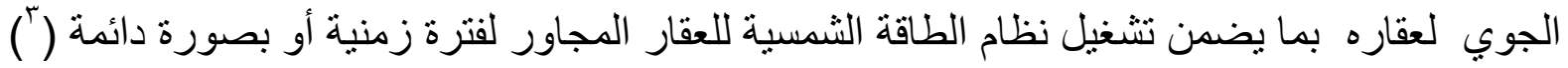

ونري أن تعريف حقوق الإرتفاق للطاقة الثمسة : هي تلك الحقوق التي بموجبها تضمن لنظام الطاقة الثمسية تلقيّ أثعة الثمس عبر المجال الجوي للعقارت المجاورة لضمان تشغيله أَّ استمرار تشغيله وعدم عرقلته بسبب ارتفاع بناء أو ظل الأشجار المحيطة و الحق في تثبت خطوط لنقل الطاقة وذللك بموجب إتفاقات طواعية والية مالم ينص القانون علي خلاف ذلك.

\section{الفرع الثالث : عناصر حقوق ارتفاق الطاقة الثمسية}

يتبين من تعريف حقوق ارتفاق الطاقة الشمسية أنها تتكون من عناصر عدة ، تتمثل في وجود نظام الطاقة

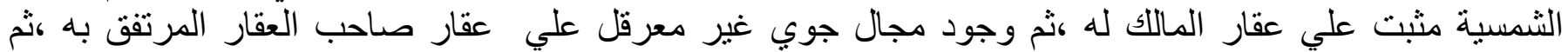

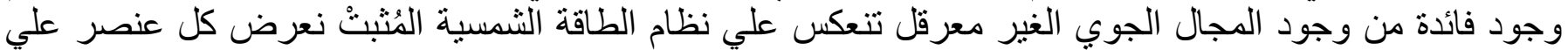
النحو التالي :

\section{أولاً :تثيت نظام الطاقة الثمسية (Solar energy System)}

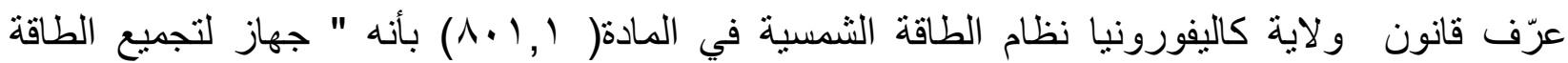

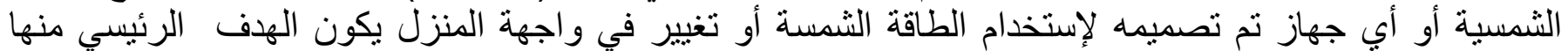

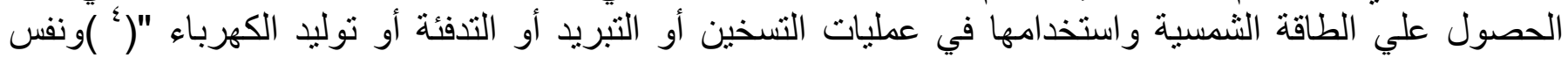
الوضع نص تشريع ولاية ويسكونسن علي أن مجمع الطاقة الشمسية هو" جهاز أو هيكل أو جزء القئ من جهاز أو هيكل

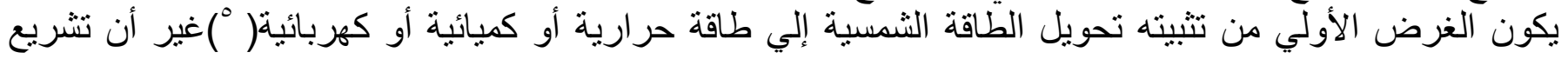

(6 )- The New Hampshire Review statute \&477,49(2017), Minnesota Statutes 2018, 500.30

SOLAR" Solar easement. "Solar easement" means a right, whether or not stated in the form of a restriction, easement, covenant, or condition, in any deed, will, or other instrument executed by or on behalf of any owner of land or solar skyspace for the purpose of ensuring adequate exposure of a EASEMENTS solar energy system as defined in section 216C.06, subdivision 17, to solar energy" Colleen McCann Kettles : Solar American Board FOR Codes and Standards Report, A:

Comprehensive Review of solar access law in the united stated, Florida solar energy Research and education foundation,2008,page 2,3.

(1)- See: Gohn wiliam.Gergacz:solar energy law : Easements of Access to sunlight,1980,p.1901

r ـ د: سمير الجمال : حقوق ارتفاق الطاقة الثمسية(دراسة مقارنة ) بحث مقدم للمؤتمر الدولي السنوي الحادي والعشرين لكلية

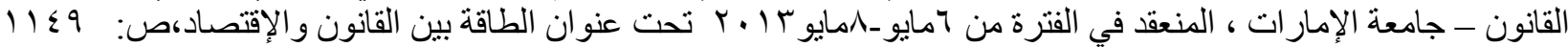

(3)- The 2018 California Act (Civil Law) , Article (801,1).

(4)- The 2018Wisconcin statutes \&Annotations,chapter700 interests in property,41 solar and wind access (2)(d)"Solar collector means a device, structure or a part of device or structure a primary a purpose of which is to transform solar energy into thermal, mechanical, chemical or electrical energy 
ولاية وايومنغ) (Wyoming statute) اعتبر المجمع الثمسي المعترف به قانوناً هو ذلك المجمع الذي الذي بكون قادراً

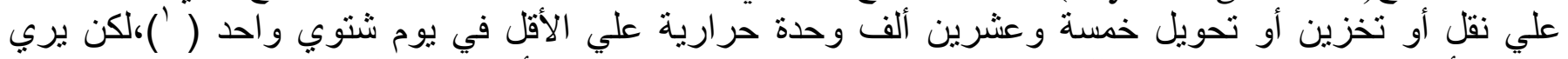

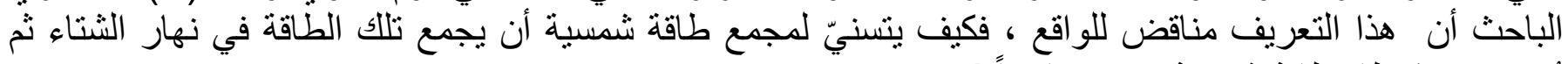

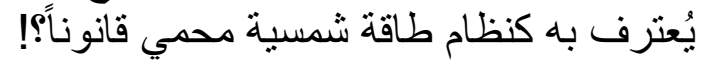

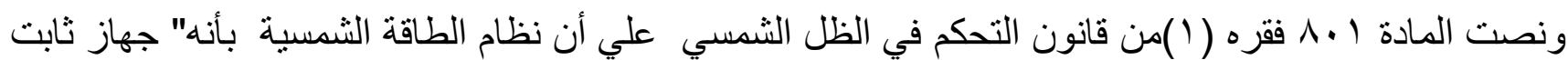

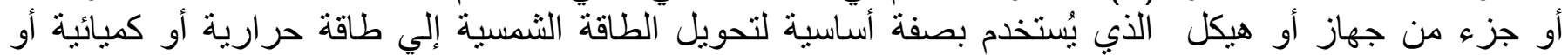

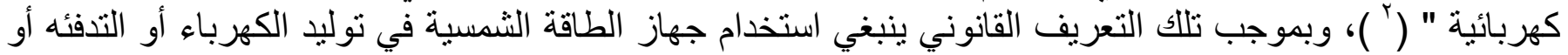

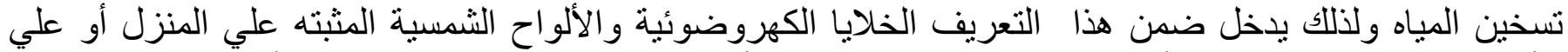

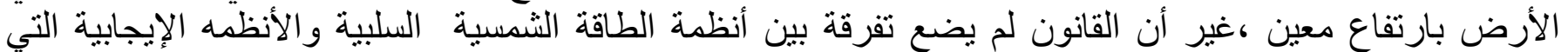

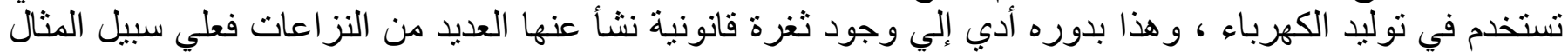

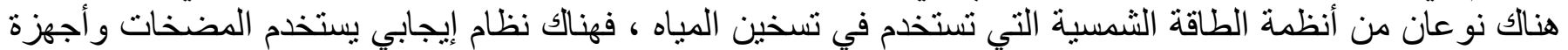

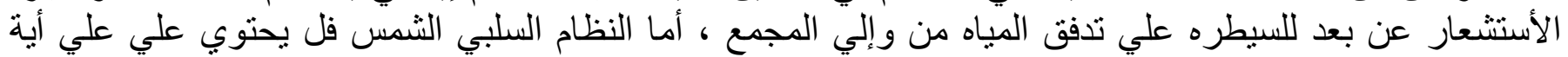

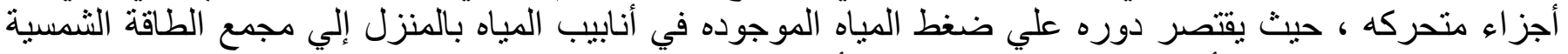

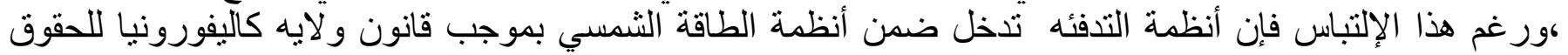

وطبقا لقانون التحكم في الظل الثمسي يجب أن يتم تثنيب نظام الطاقة الثمسية علي المنزل أو المبني بواجها

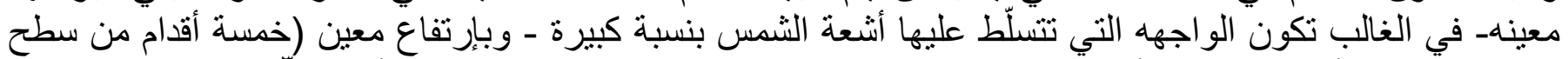

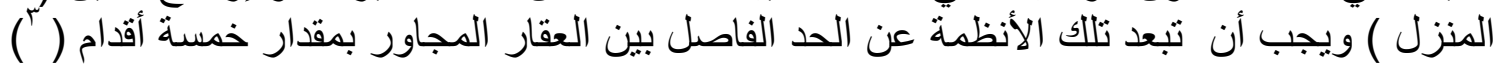

\section{ثنانيا : وجود مجال جوي غير معرقل) Airspace Without Obstruction)}

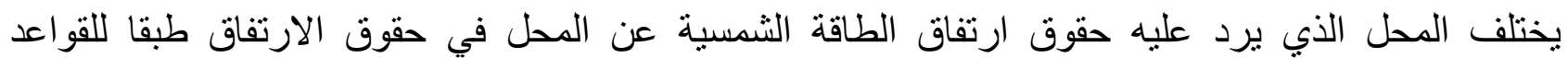

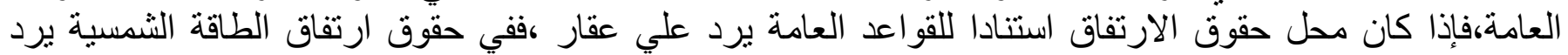

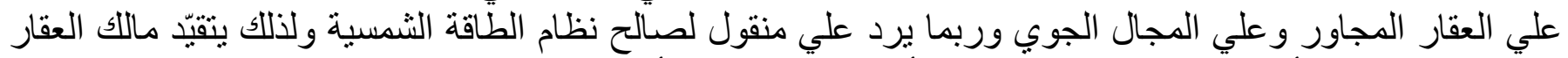

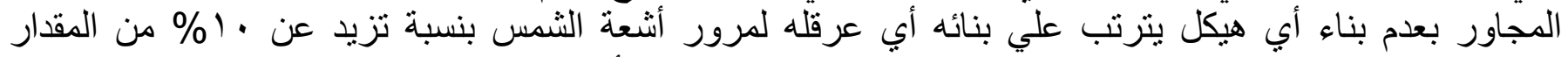

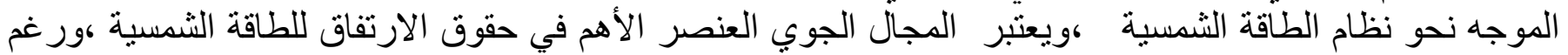

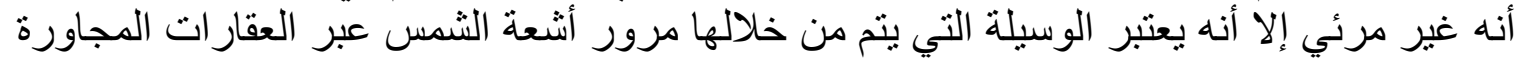

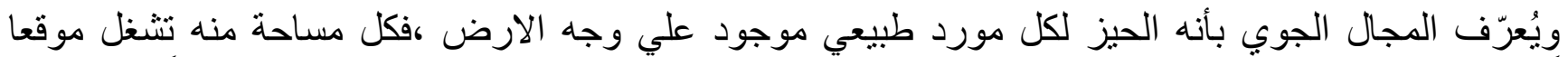

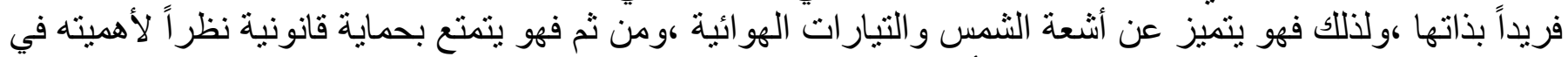

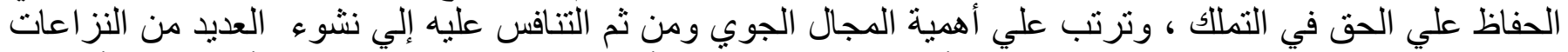

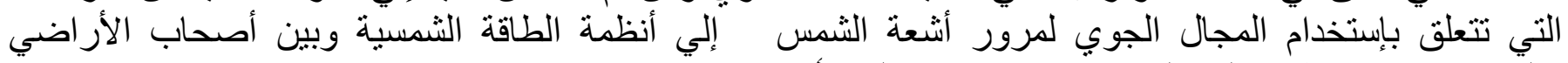

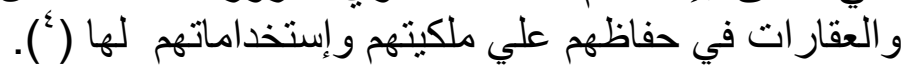

1)- TheWayomig STAT, title34- PRPERTY CONVEYANCES AND SECURITY

TRANSACTIONS CHAPTER 22- SOLAR RIGHTS 102."

(2)- The2018 Solar Shade Control Act

(3)- The 2018California Solar Shade Control Act

(4)- See:RULE, Troy "Airspace in a Green Economy" Law Review Journal,Vol:59, school of law ,University of Missouri ,(2011).p275. 


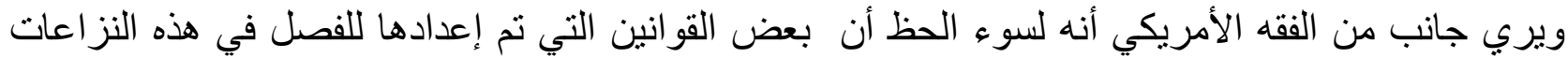

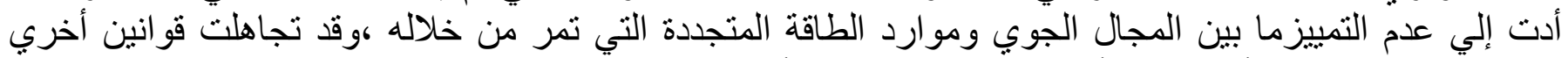
حقوق المجال الجوي لأصحاب الأراضي والعقار ات من أجل تفضيل إستخدامات المجال الجوي الخاصة التي تدعم

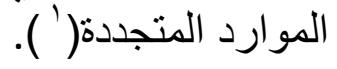

وجدير بالذكر أن إستخدامات المجال الجوي في السنوات الماضية كانت إستخدامات لاينشأ عنها إلا نزاعات

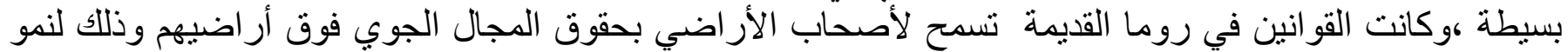

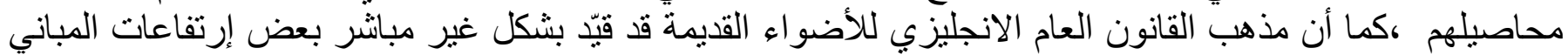

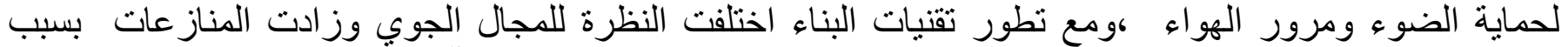

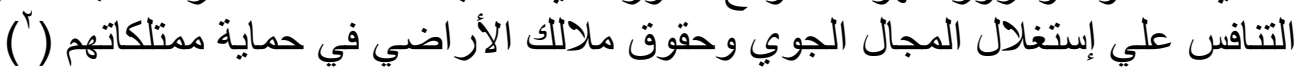

وقد دفعت الأهمية المتزايدة للمجال الجوي الحضري الناشئة عن تقنيات البناء الثاهقة الارتفاع إلي زيادة تحديد حقوق المجال الجوي بين أصحاب الممتلكات بسبب الزيادة المطردة في عدد الهياكل المنفردة التئ التي تحتوي

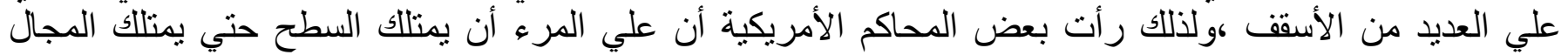

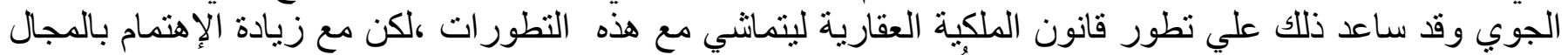

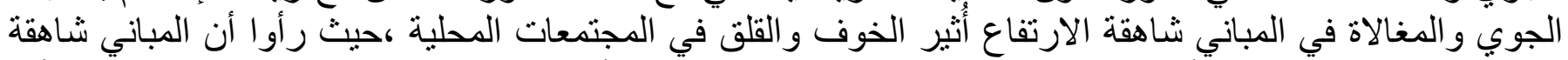

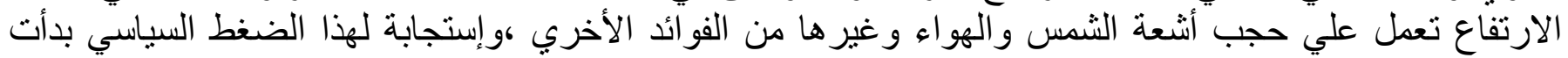

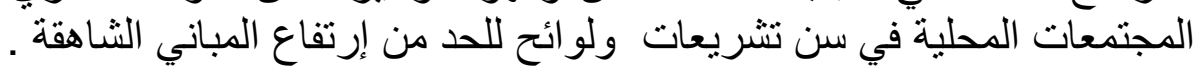

و علي الرغم من أن قيود الارتفاع تدفع ملاك الأراضي إلي التخلي عن حقوقهم في شغل المجال الجوي إني

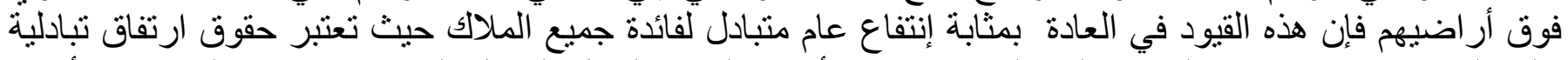

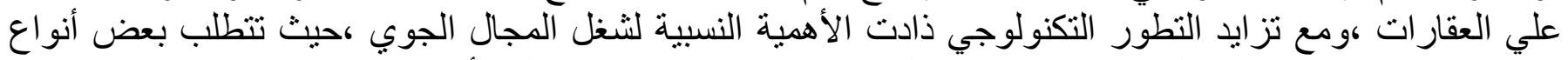

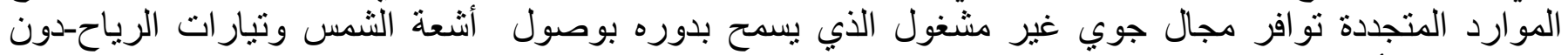

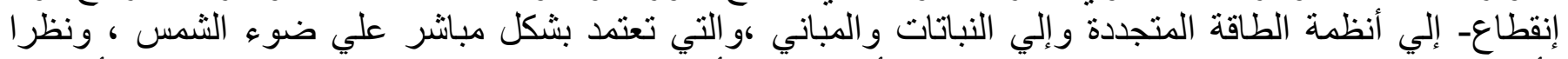

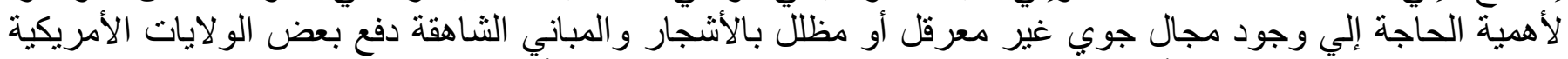

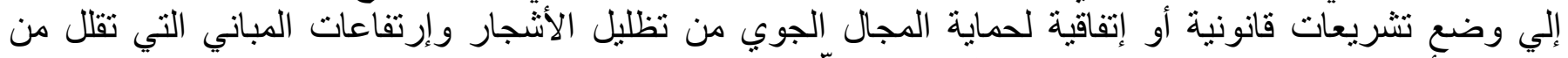

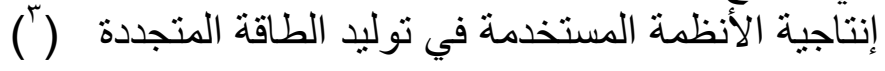

ثالثاً :يجب أن يكون هناك فائدة تتعكس علي نظام الطاقة الشمسية

(1)- Rule troy :Ibid,(2011)p:279

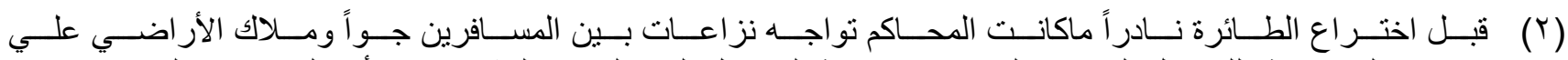

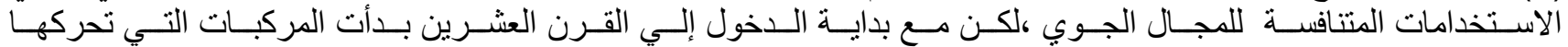

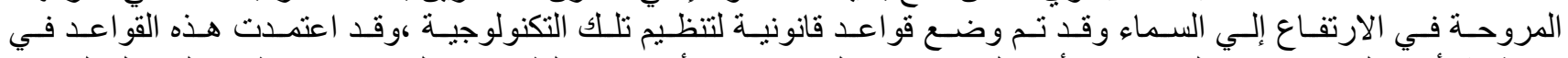

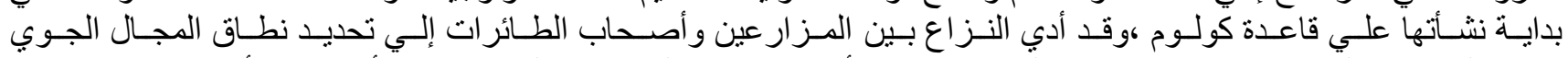

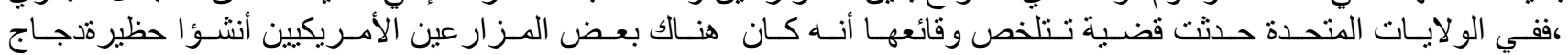

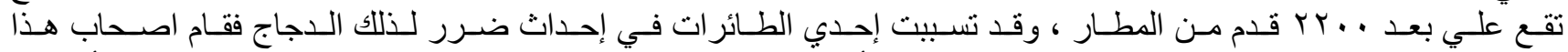

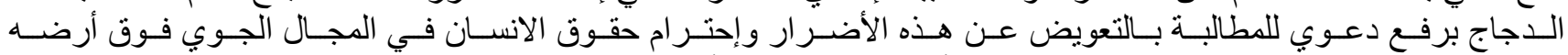

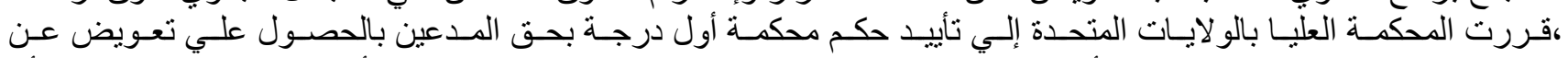

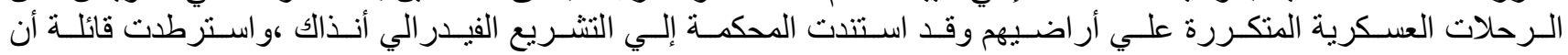

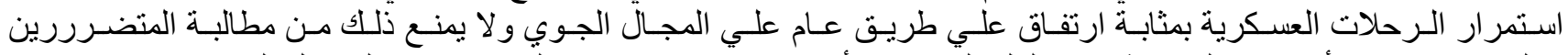

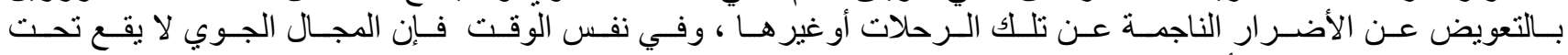

See :Troy, A. op. cite(2011), p:176. السيطرة الحصرية لملاك الأر اضي والنيـ المز ارعين. (َّ) -المرجع السابق :دسمير حامد الجمال ، "حقوق ارتفاق الطاقة الثمسية"، ص: 


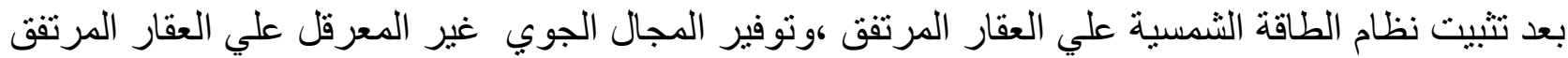

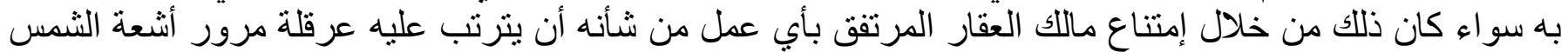

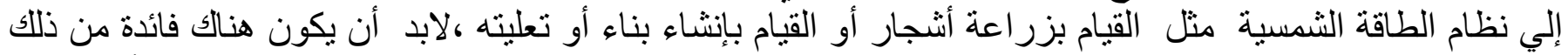

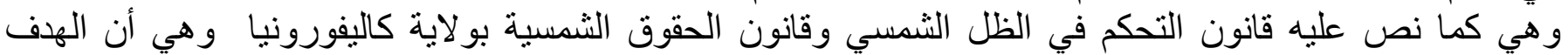

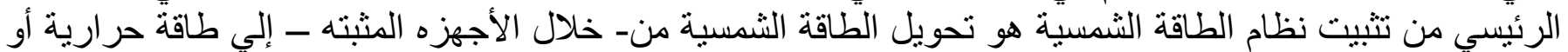

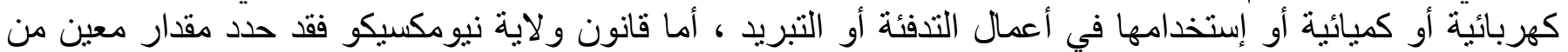

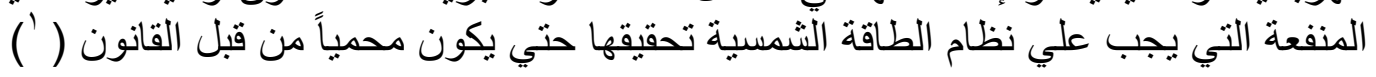
يتضح مما سبق ذكره أن عناصر حقوق ارتفاق الطاقة الثمسية هي وجود نظام طاقة شمسية مثبت علي

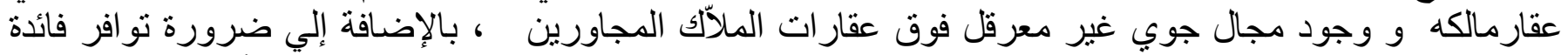

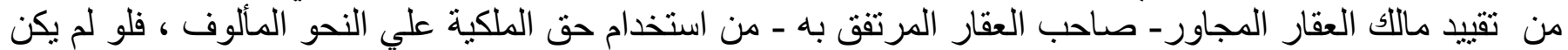

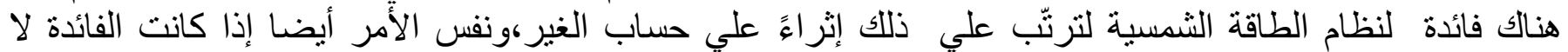

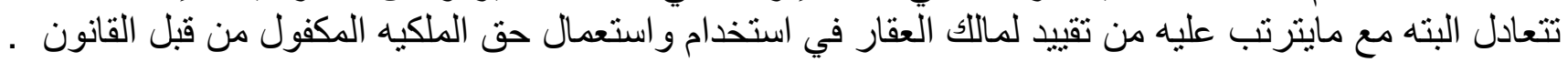

\section{الفرع الرابع :خصائص حقوق ارتفاق الطاقة الثمسية}

تتفق خصائص حقوق ارتفاق الطاقة الثمسية مع حقوق الارتفاق العامة في كونها حقوق ارتفاق سلبية كومتفر عة عن حق الملكية علي النحو التالي :

\section{أولا: حقوق الارتفاق الطاقة الثمسية حقوق ارتفاق سلبية}

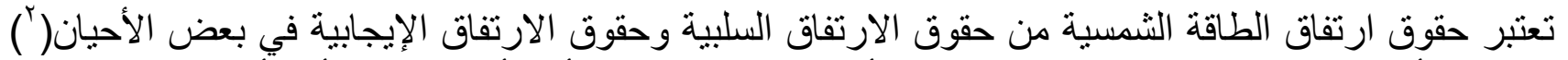

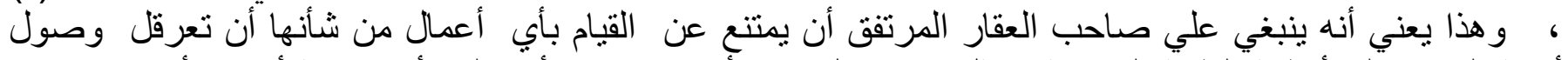

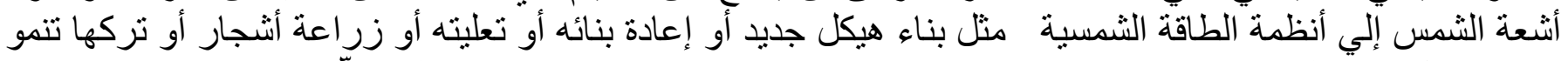

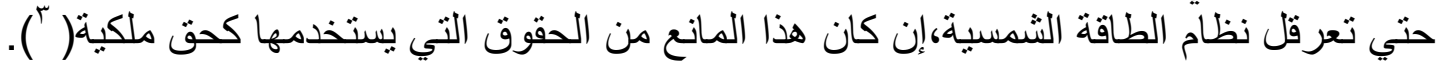

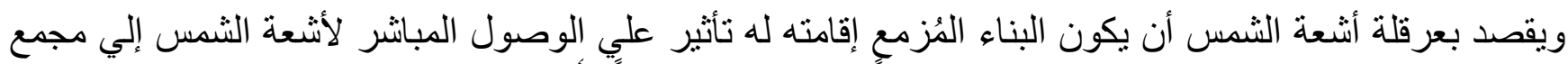

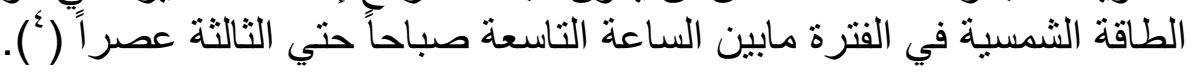

وبطبيعة الحال كلما كانت أنتعة الثمس مُسلّطة بصورة مبانشرة نحو مجمع الطاقة الثمسية كلما زاد من إمكانية

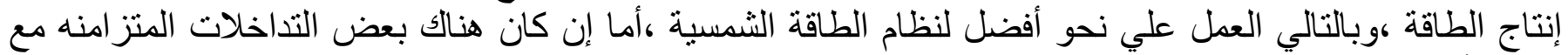

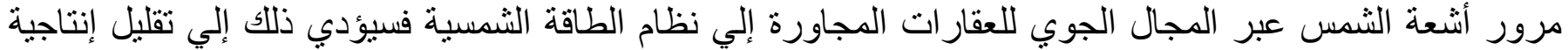
الطاقة المولدة من نظام الطاقة الثمسية ( ) ثانيا : حقوق ارتفاق الطاقة الشمسية متفرعة عن حق الملكية :

(1)- The 2009New Mexico, state 47-3-34, (updates 2017)

(r) -و يتعلق ذلك بالمشروعات الكبيرة التي يتطلب فيها مساحات واسعة من الاأراضي ، حيث يشترط توافر طرق للوصول إلي

(3)- William.a.john.op.cite.p:129. الموقع أو الطريق العام ومرور المعدات اللازمة.

(4)-Wis. State 2011( Updates 2017)

(5)- Colleen MCcann, kettles:Ibed:3 - 4 


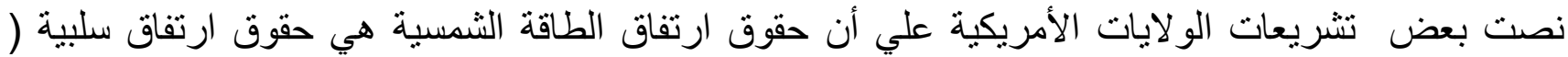

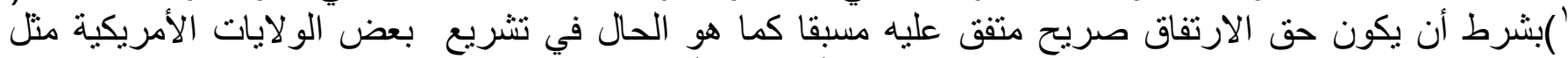

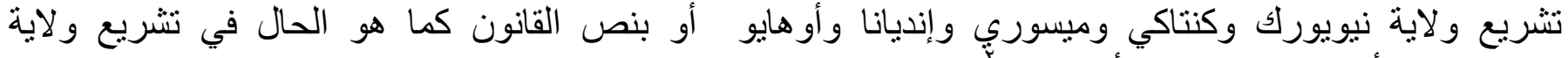

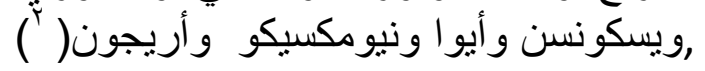

\section{المطلب الثاني : :الأساس القانوني لحقوق ارتفاق الطاقة الثمسية}

يعتمد التكييف القانوني لحقوق الارتفاق ـوفقاً للقواعد العامة-علي كونها حقوق عينية تابعة لحق الملكية

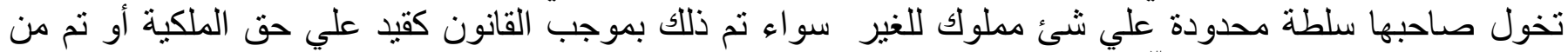
خلال المالك الأصلي للعقار (") ،وحتي يتم تحديد التكييف القانوني لحقوق الارتفاق الطاقة الثمسية أليس من باب أولي تحديد التكييف القانوني لأنظمة الطاقة الثمسية ؟ وفي أبي حقل قانوني تقع أهي منقول أم عقار أم عقار

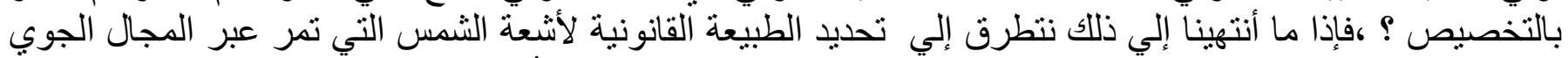

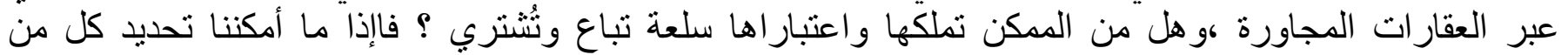

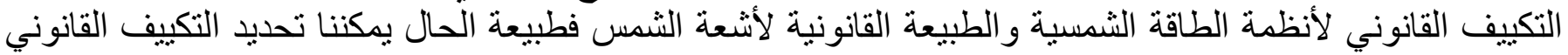
لحقوق ارتفاق الّاقة الثمسية كل في فرع مستقل علي النحو التالي:

\section{الفرع الأول : التكييف القانوني لأنظمة الطاقة الثمسية}

لم يشر المشرع الأمريكي أو المصري- صر احةًَ للتكييف القانوني لأنظمة الطاقة الثمسية وبالتالي بدفعنا إلي

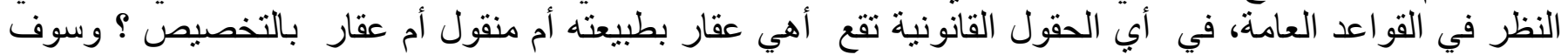

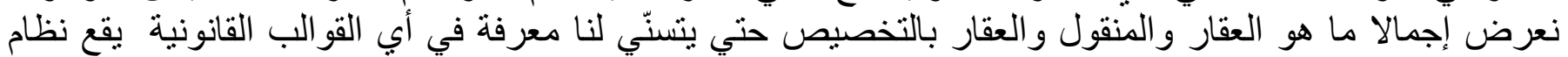
الطاقة الثمسية

\section{أولا: العقار بطبيعته :}

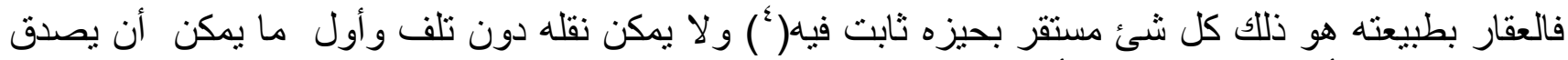
علي ذلك هو الأرض التي تعتبر الأصل في كل عقار ، فهي التي لايمكن نقلها من مكان إلي آخر إلا من خلال

(6)- Wiliam.a.john.op .cit .p:132

(1)- The New Mexico . Stat .\& 47-3-4,to 47-3-9)(2009)(updates2017) the2017 Oregon Revised Statutes Volume : 03 - Landlord-Tenant, Domestic Relations, Probate Chapter 105 - Property Rights Section 105.890 - Solar energy easement appurtenant" (1) A solar energy easement shall be "appurtenant to and run with the real property benefited and burdened by such an easement

( ب) ـ لمزيد من التفاصيل عن التكييف القانوني لحقوق الارتفاق راجع : د.مقلاني مني، النظام القانوني لحق الارتفاق في التشريع

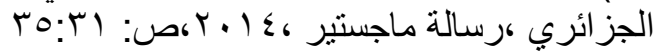

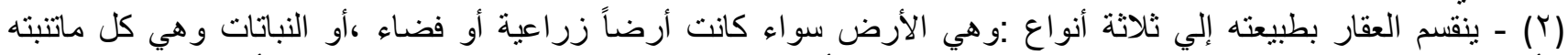

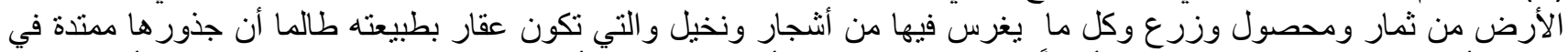

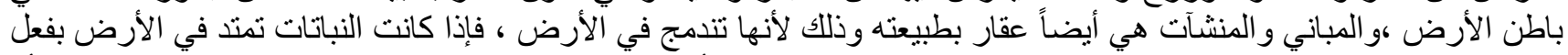

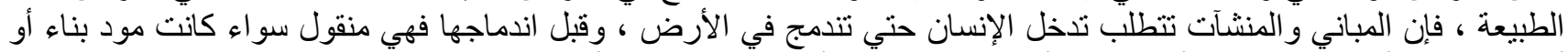

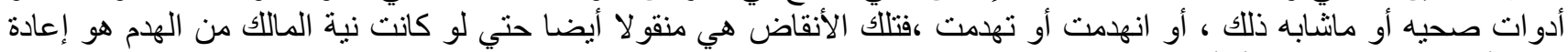

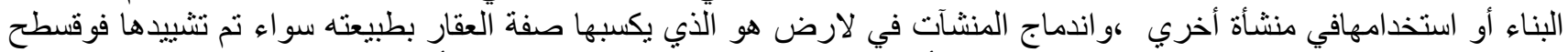

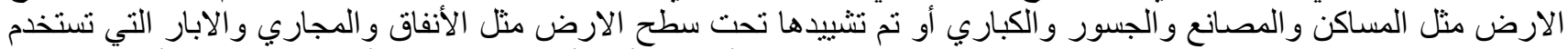

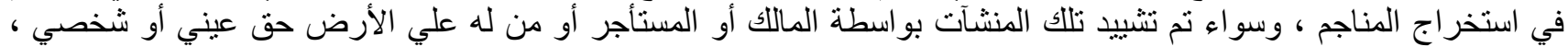

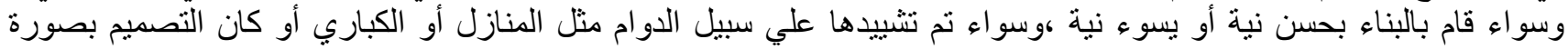


أجز اء من الأرض أو من خلال فقد ذاتيتها وتحولها إلي منقول منذ إنتزاعها من الأرض ونقلها إلي مكان آخر

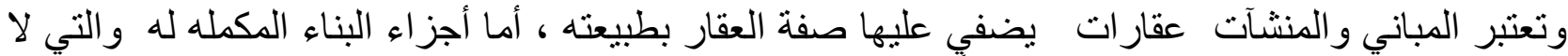

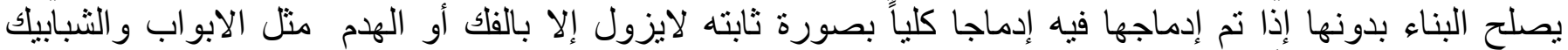

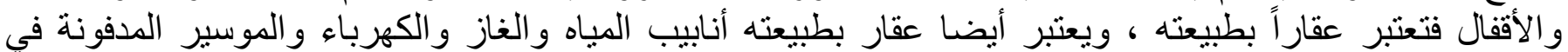

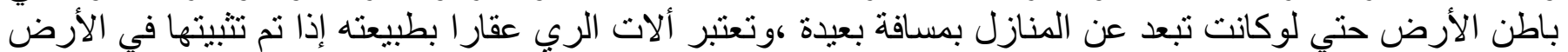
بحيث لا يمكن فصلها إلا من خلال فلك جميع أجز ائها

ثانيا :العقار بالتخصيص :

يُعرّف العقار بالتخصيص بأنه المنقول بطبيعته الذي يضعه المالك لخدمة عقاره أو إستغلالكة( ) و الجدير بالذكر أن فكرة العقار بالتخصيص لم تكن من ابتداع المشرع المصري و إنما نقلها عن المشرع الفرنسي

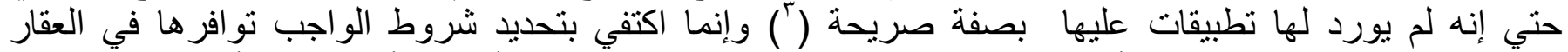
بالتخصيص وهي اتحاد المالك وأن يكون العقار بالتخصيص قد ارتبط بآلعقار الأصلي لأجل خدمته أو استغلالّه ، فأما عن شرط إتحاد المالك فتم استخلالصه من المادة rي من القادئ القانون المدني التي اعتبرت العقار بالتخصيص

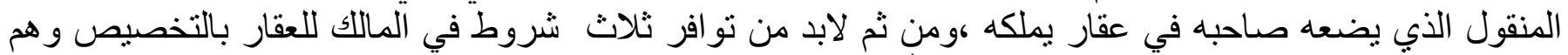

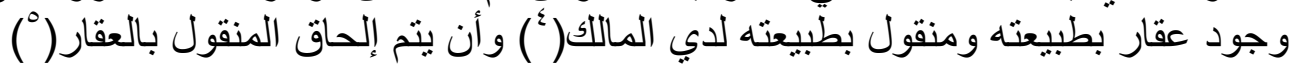

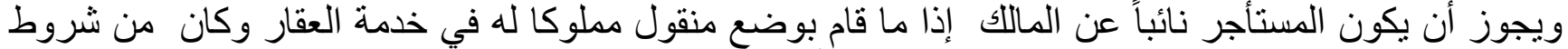

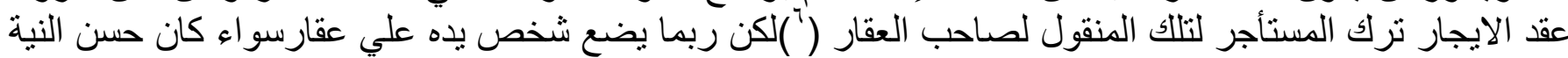

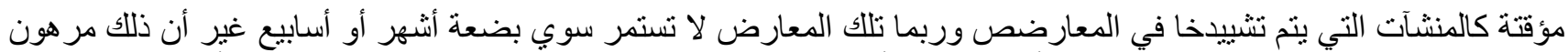

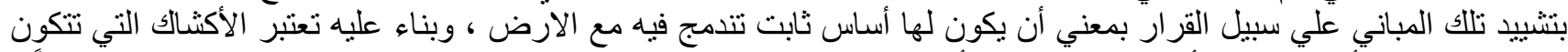

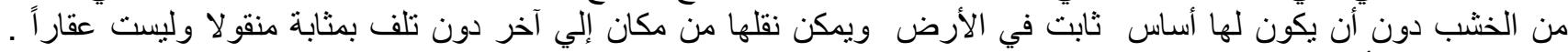

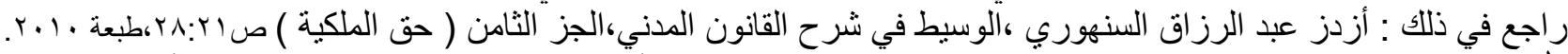

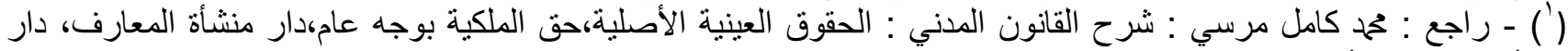

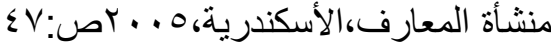

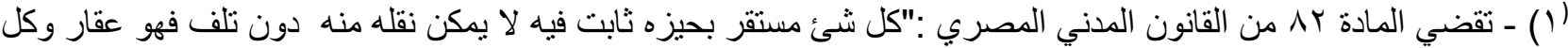

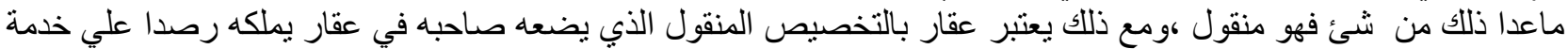
هذا العقار أو استغلالهل

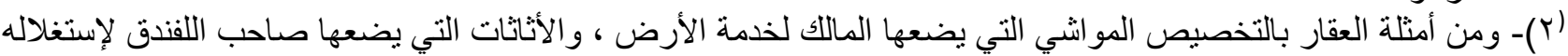

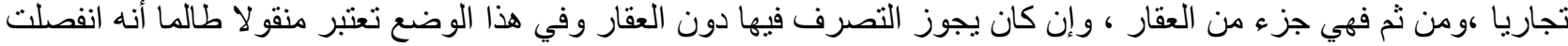

$$
\begin{aligned}
& \text { عن العقار دون تلف . تجائ }
\end{aligned}
$$

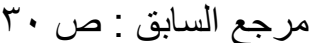

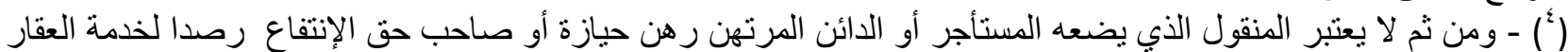

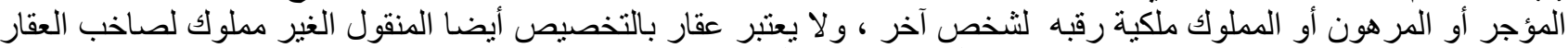

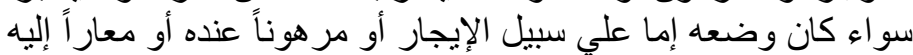

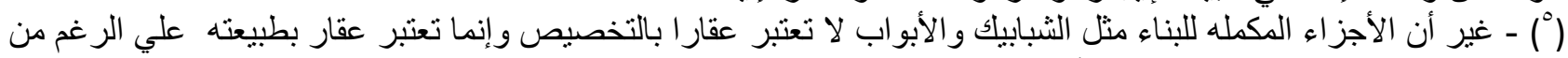

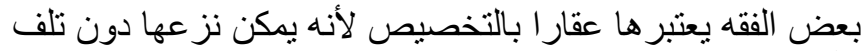

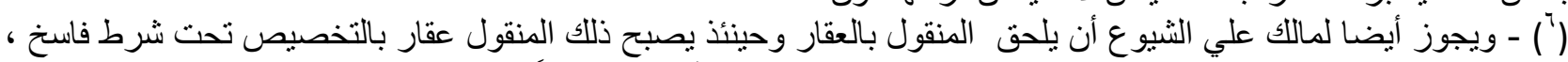

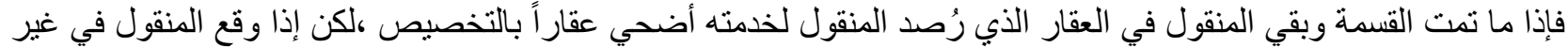

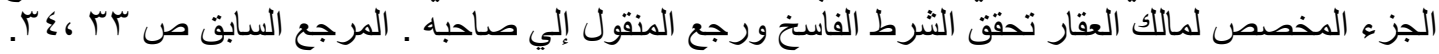




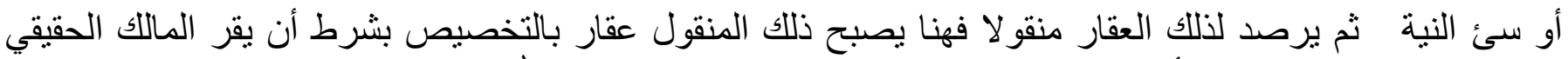

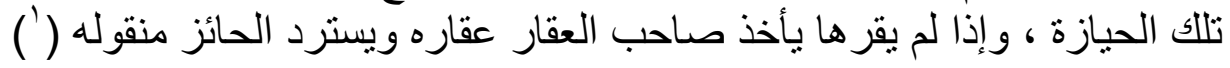

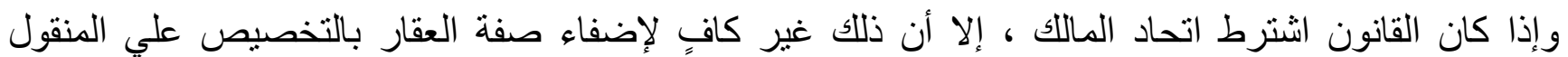

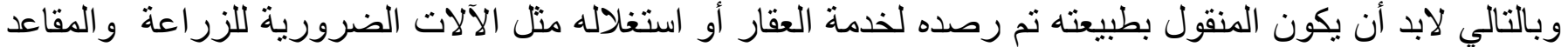

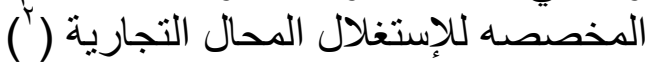

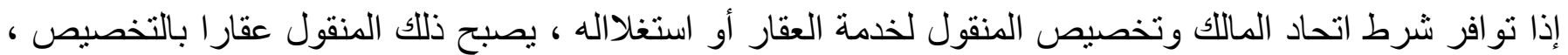

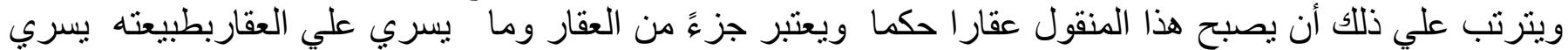

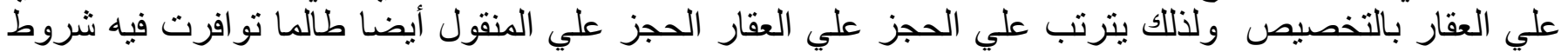
العقار بالتخصيص وما يسري علي العقار من رهن أو حق اختصاص يسري علي المنقول أيضا لئا لئا

ثالثا : المنقول :

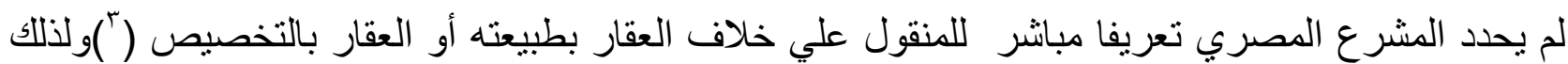

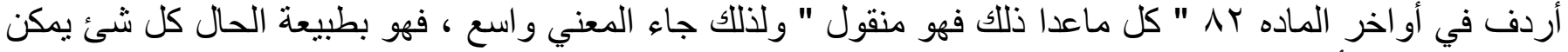

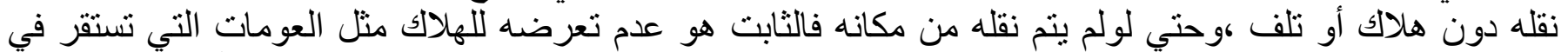

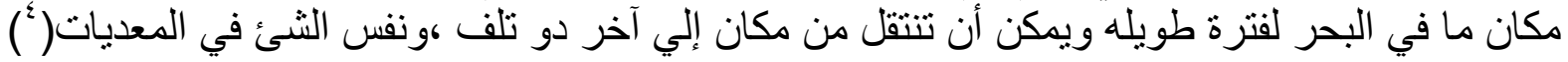

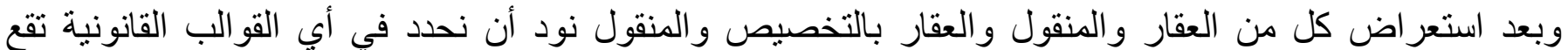

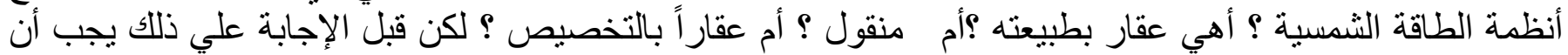

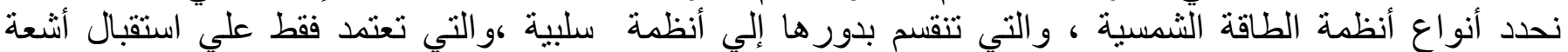

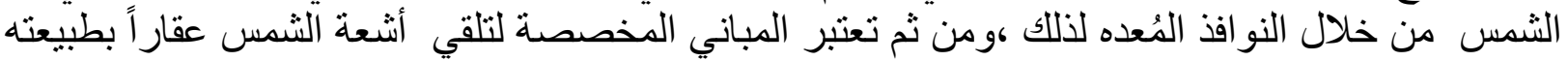

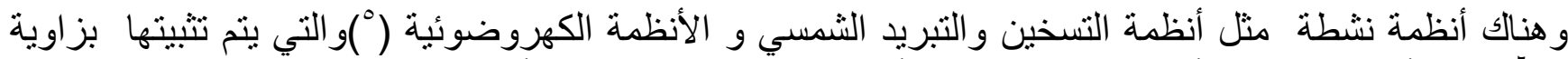

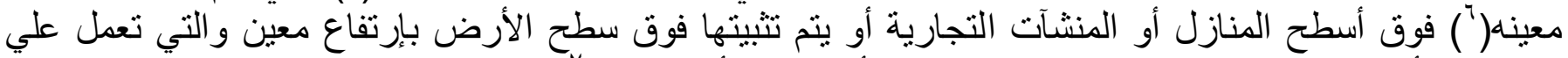

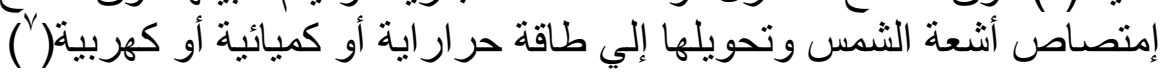

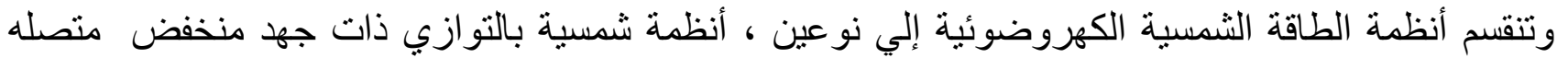

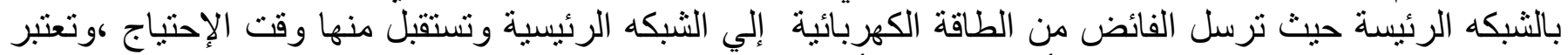

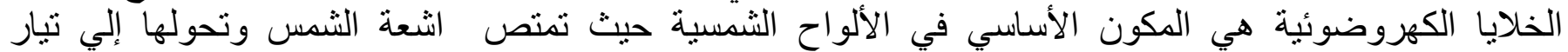

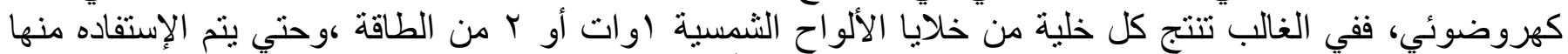
علي النحو الأمثل يتم ربط مجموعه من الخلايا ربطا محكماً لتتنج أكبر قدر من الطاقة ،فربما تنتج لوحه شمسية الطية

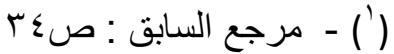

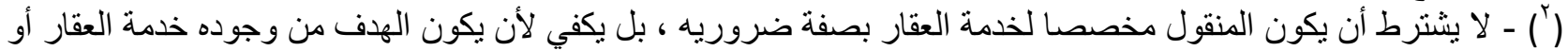

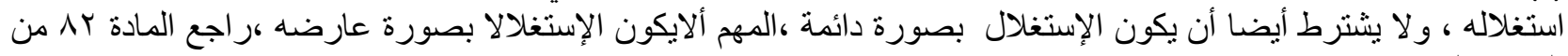

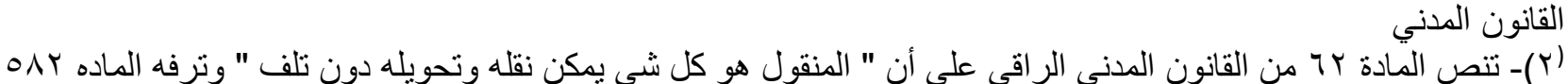

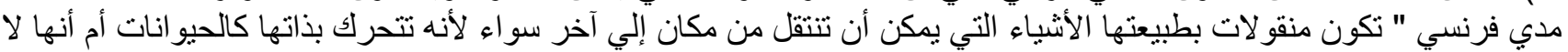
تسنطيع أن تغير مكانها إلا بتأثير قوة أجنيبة كا الأثياء الحية.

(4)-The 2018 California solar shade control act.

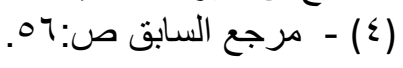

(" (")- يجب علي مستخدم الطاقة الثمسية الإستعانه بفني عند تثثيت وتثغيل أنظمة الطاقة الثمسية كما أن دوره بستمر في عملية الصيانه (V) - راجع في ذلك : الاليل الإرشادي لنتبيت أنظمة ألواح الطاقة الثمسية الكهروضوئية بإمارة أبو ظبي علي الموقع التالي:WWW.rsb.gov.ae - تاريخ الزياره 


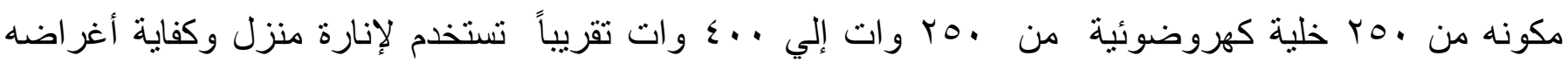
بالكامل من

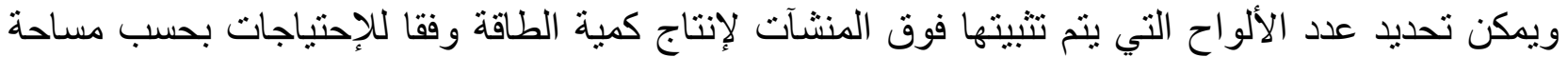

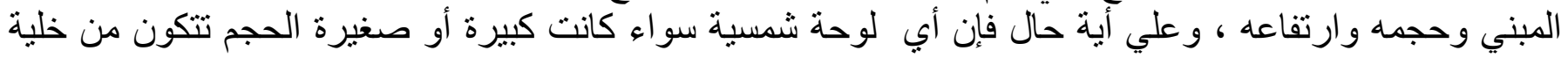

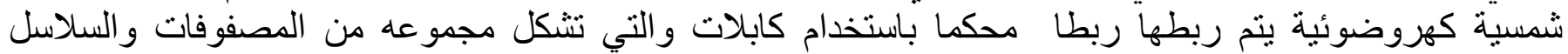
كماتتكون من مجمو عه من المفاتيح الكهربائية و الهياكل الميكانيكية التي تستخدم في تثبيت الألواح الثنمسية وتوجيهها

نحو أثعة الثمس (')

وبعد استعر اض أنظمة الطاقة الثمسية النشطة يتضح - بحكم القواعد العامة ـ أنها تقع في دائرة العقار

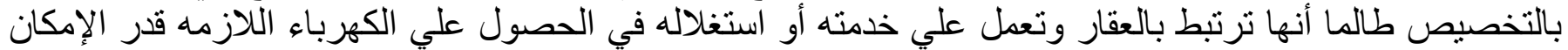

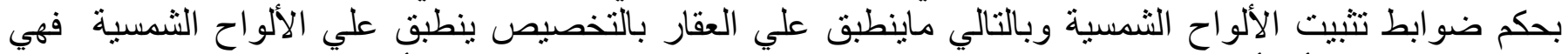

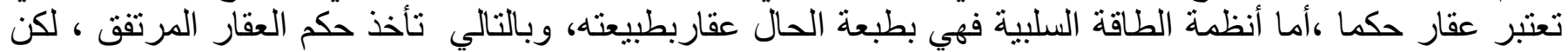

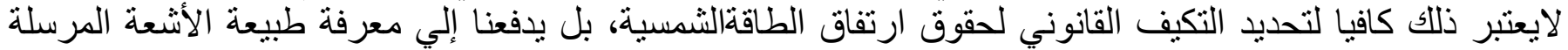

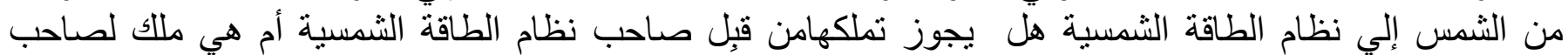
العقار المرتفق؟ أم هي بمثابة حق طبيعي لا يجوز الإستحواز عليه ؟ و ولي أي مدي يكون ملكية المجال الجوي ؟هذا ما سنحدده في المطلب التالي

\section{الفرع الثاني : الطبيعة القانونية لأشثعة الثمس والمجال الجوي}

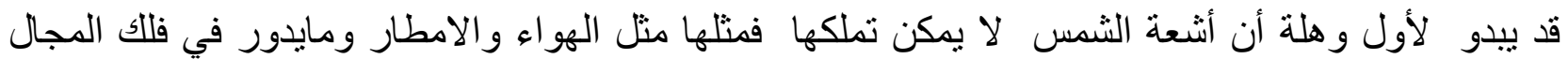

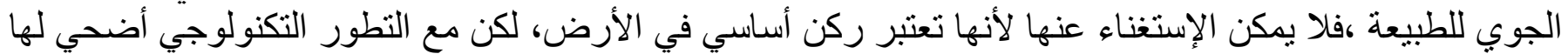

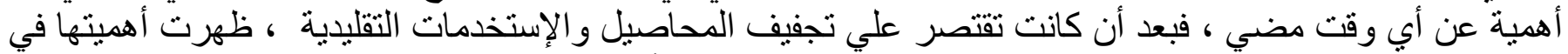

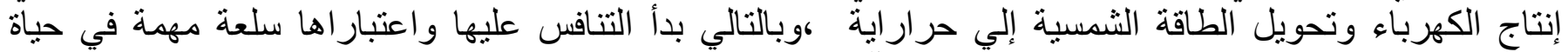

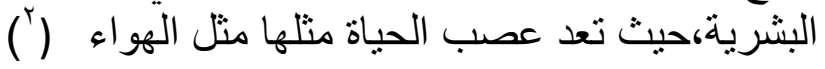

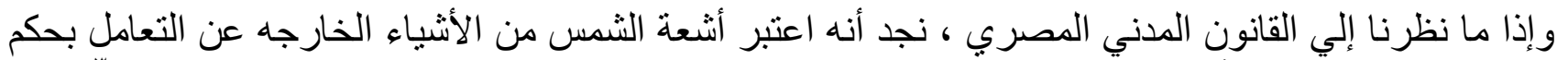

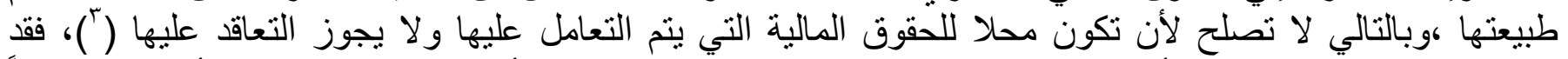

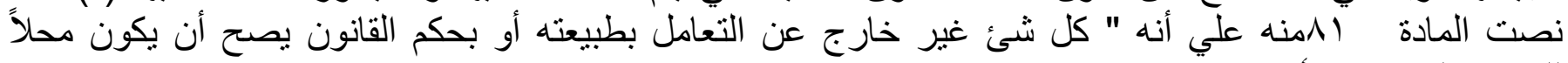
للحقوق القانونية "( أ)

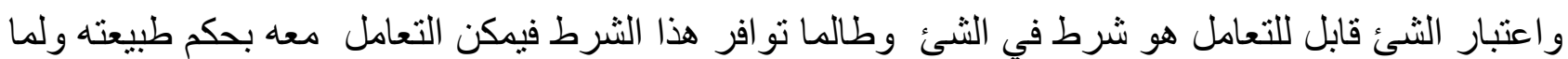

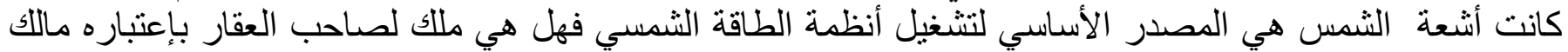
المجال الجوي أم تعتبر من الأثشياء الخارج التعامل عليها؟

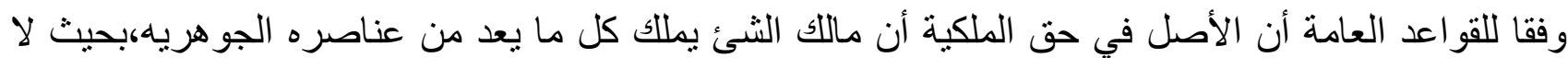

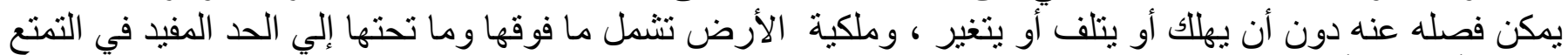

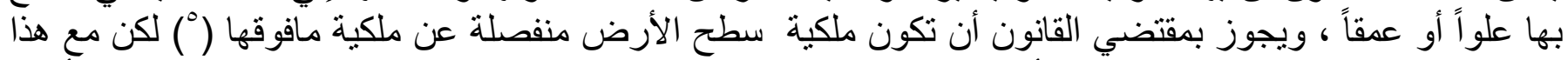

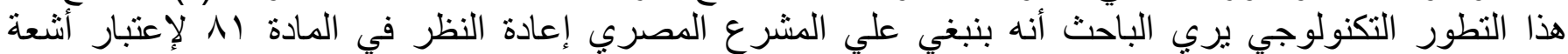

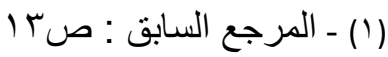

(1)-See: Reize ,G.L."A solar rights zoning guarantee seeking new law in old concepts" , ,law ReviewJOURNAL N:3, Washingoton University (1976),p:377:379

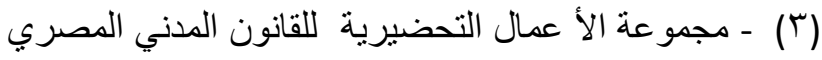

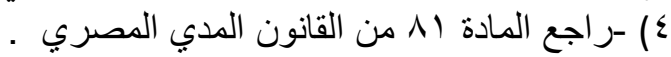

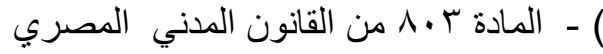


الثمس من الأشياء التي يتم التعامل عليها كأي سلعة تباع وتشتري ويتم نقلها مثلها مثل الكهرباء ،كما ينبغي تعديل

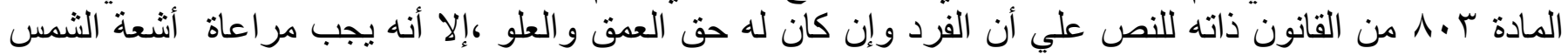

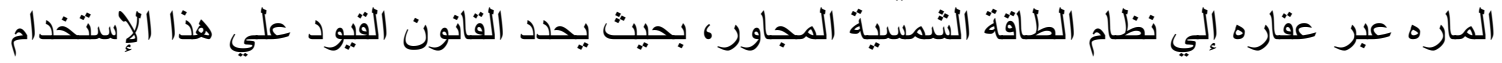

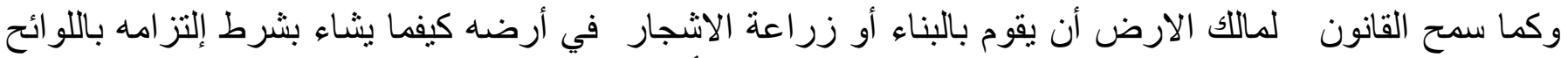

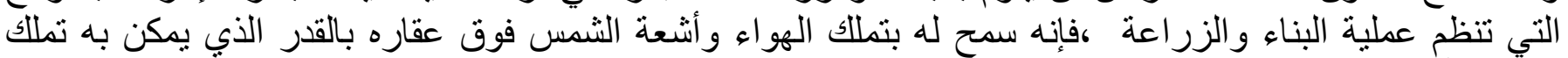

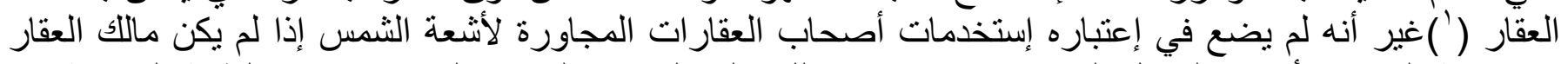

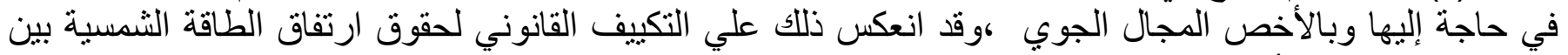

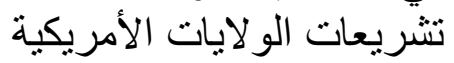

\section{القرع الثالث:التكييف القانوني لحقوق ارتفاق الطاقة الثمسية}

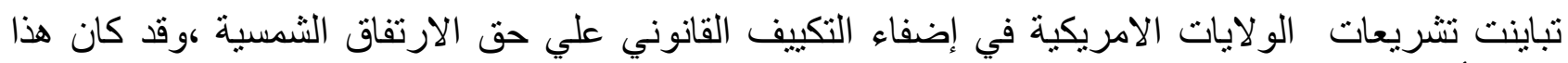

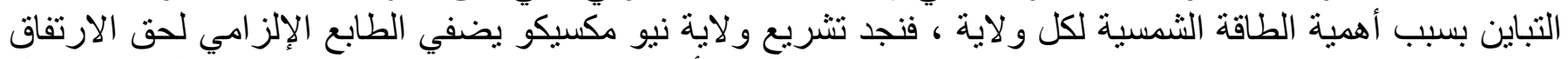

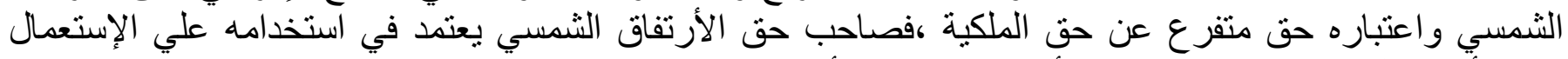

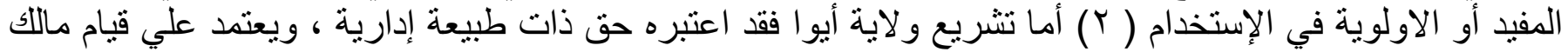

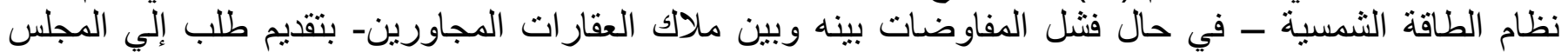

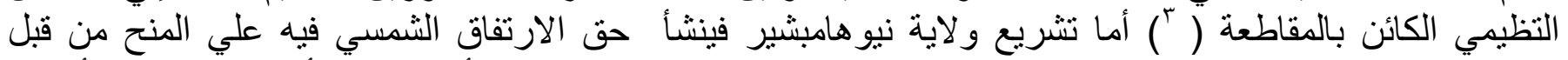

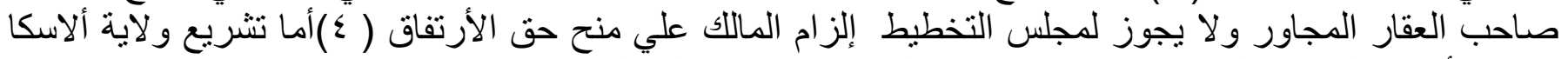

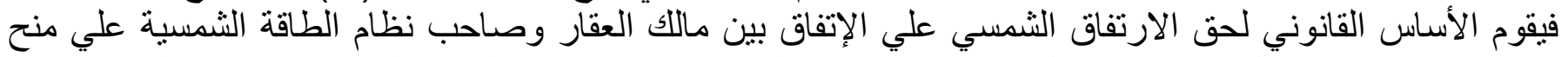

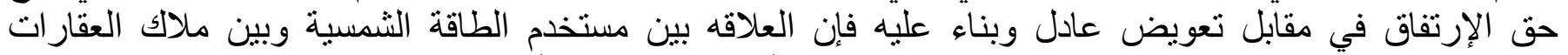

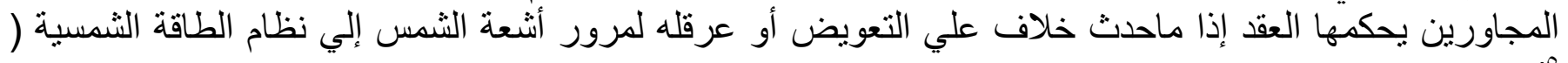

أماحق الارتفاق الثمسي في تثريع ولاية ولاية كاليفورونيا فذو طبيعة مزدوجة ، فإذا كان حق الارتفاق

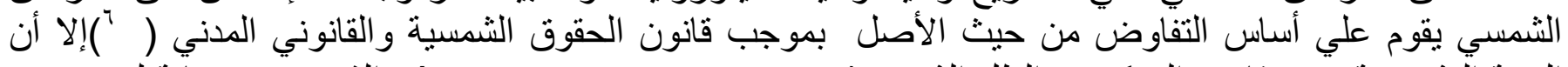

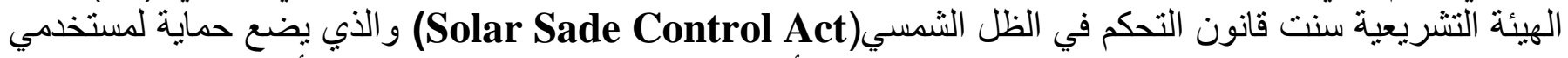

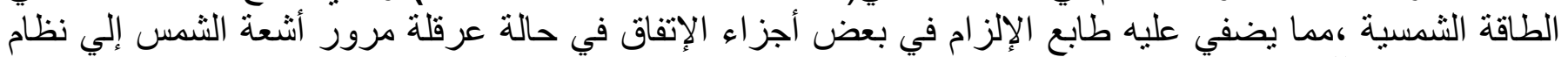

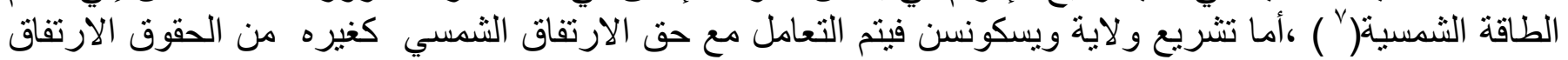

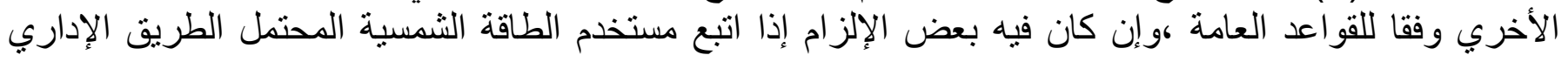

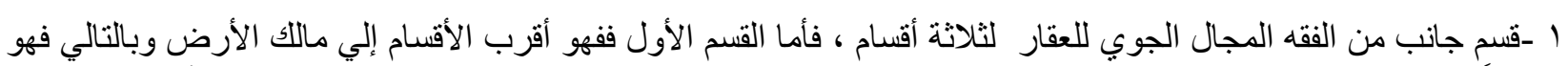

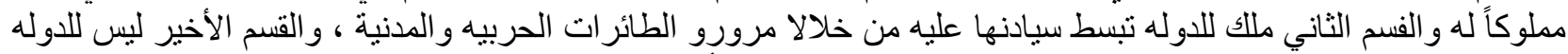

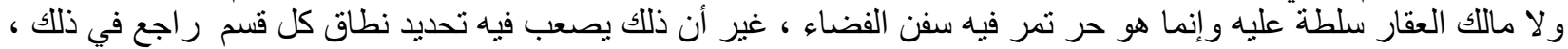

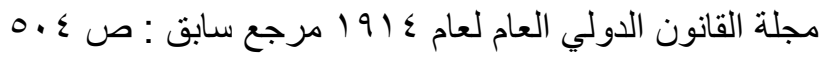

(2)- THE New Mexico statute ,47-3 1t0 47-3-5(2017)

(3)-The Iowa Code ,ChpTER 56-4 (a ) (2014)(Updates 2017)

(4)- New Hampshire 477-50 (2018)

(5)- The Alaska code state Ann,34-15-145(A)(3)(2018)

(6)- The CaliforniaAct( Civil Code)(704,1)(1978)Updates civil code (2018)

(1)- The 2018 California Solar Shade Control Act 


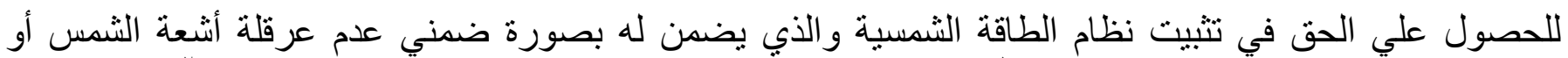

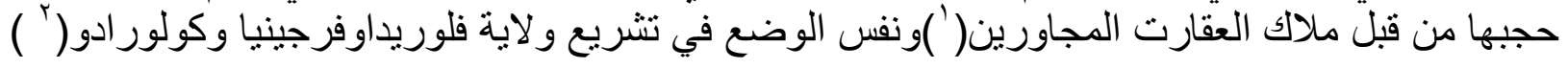

\section{الخاتمة}

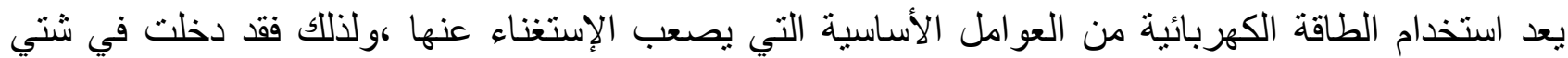

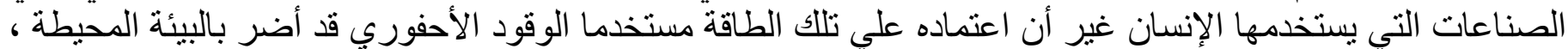

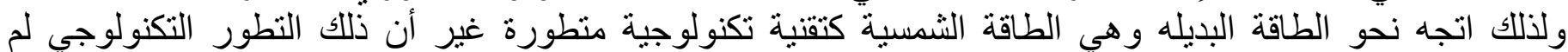

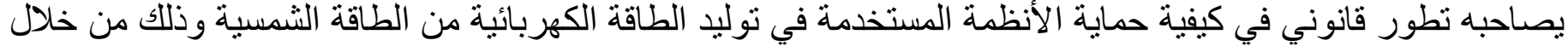

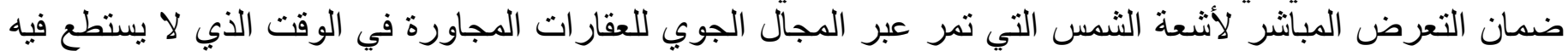

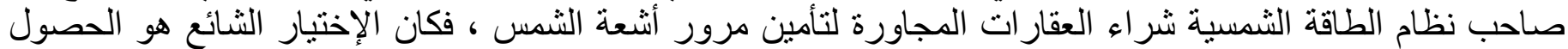

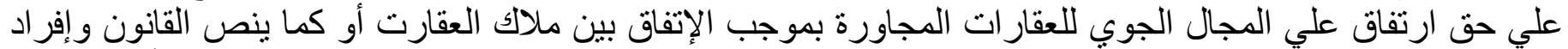

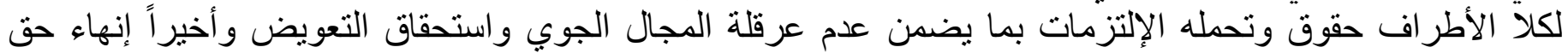

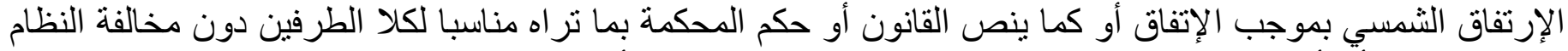

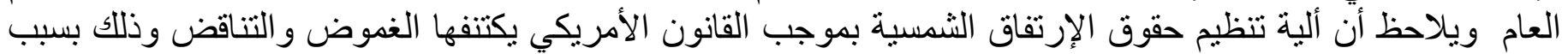

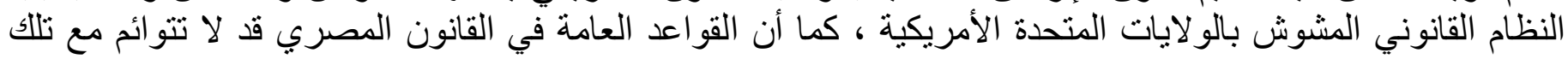

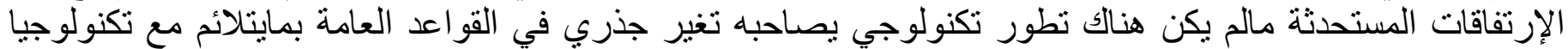
الطاقة الثمسية الثان الثية

\section{التوصيات : (1)}

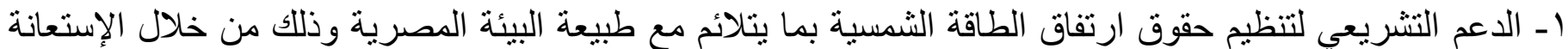

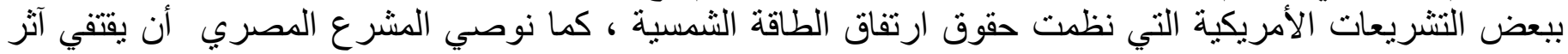
تشريع و لاية كاليفورونيا ويسكونسن في تنظيم حقوق ارتفاق الطاقة الثنمسية

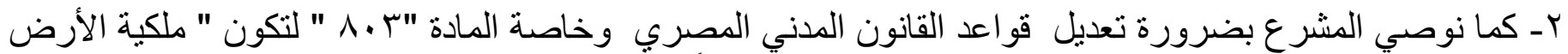

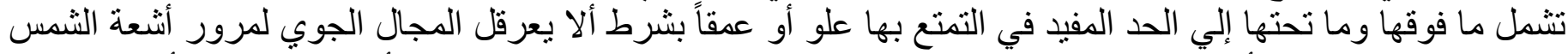

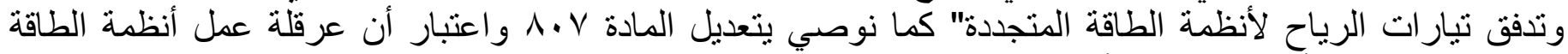

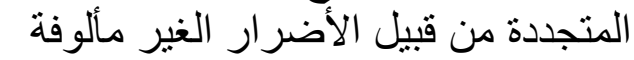

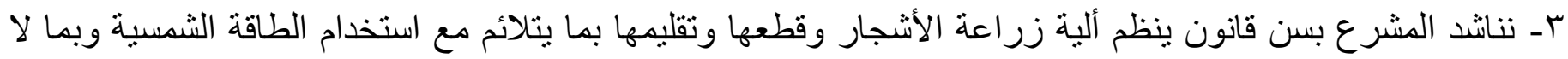

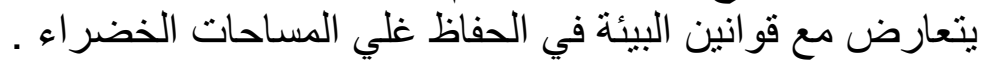

كـ ضرورة اعتبار أن تثبيت نظام الطاقة المتجددة علي الأراضي أو فوق العقارات من القواعد التي يتعلق بالنظام العام

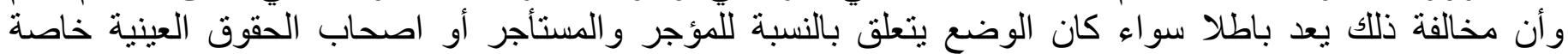
صاحب حق الإنتفاع .

عـ- الدعم الإداري للجهات المختصة بإصدار تصاريح الطاقة الثمسية من خلال توفيرمختصين لديهم خبرة وكفاءة بما

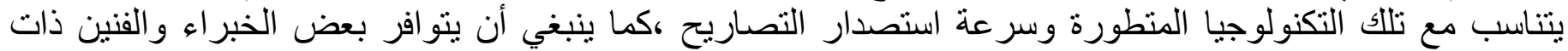

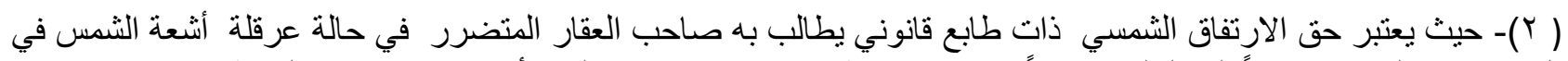

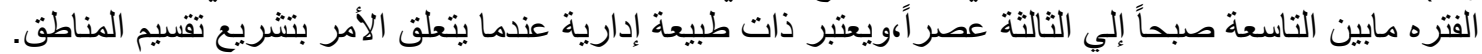

(3) The Wisconcin stat,700,35 (2018) The Florida State, Code - Real and personal property ,easements -chapter 704,07), The Virginia Code -55-35-4 (2017),THE Colorado ,Revised statutes (,Title 38- Property - real and personal property (5-101)(2017) 


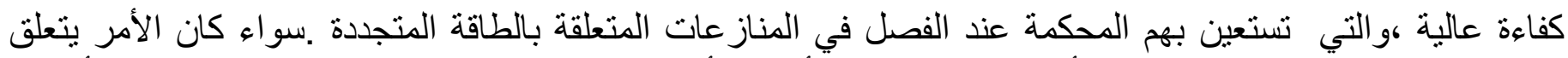
بين مستخدمي الطُّة المتجددة وبين أصحاب العقارت ، أو بين أصحاب مشرو عات الصات الطاقة المتجددة فيما بينهم أو بين الدولة . لبن

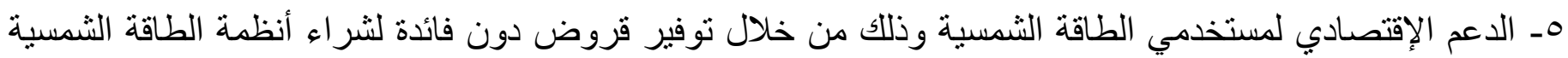

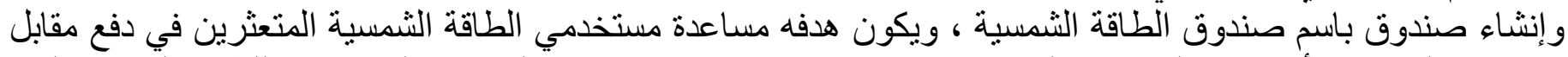

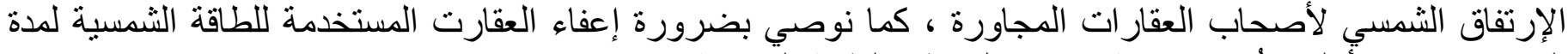

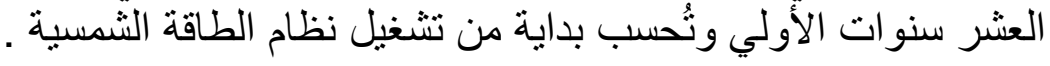
الـ الدعم الإعلامي بضرورة توعية المواطنين بأهمية الطاقة الثمسية وتبصير هم بفوائدها و أضرارها لترسيخ فكرة

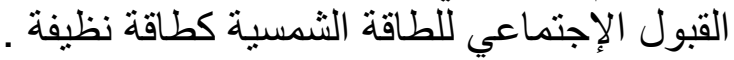




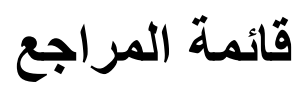

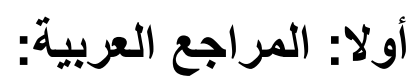

1-- د: سمير الجمال : حقوق ارتفاق الطاقة الثمسية(در اسة مقارنة ) بحث مقدم للمؤتمر الدولي

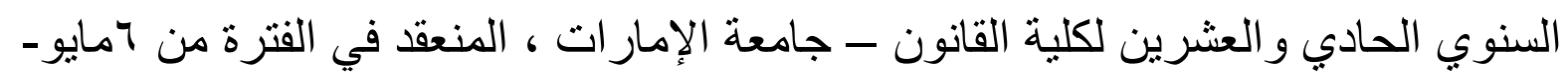

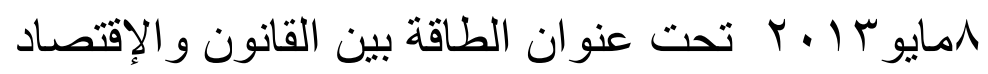

ץ-.مقلاني مني، النظام القانوني لحق الارتفاق في التثريع الجزائري ،رسالة ماجستير(ع ( ب )

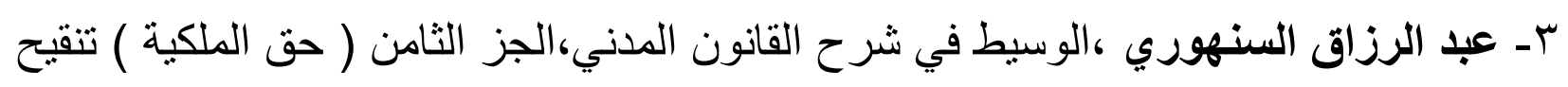

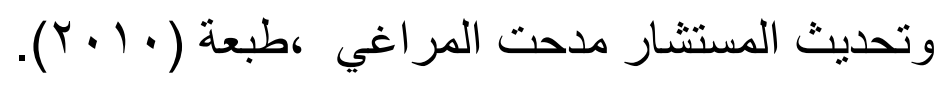

ع - محم كامل مرسي : شرح القانون المدني : الحقوق العينية الأصلية،حق الملكية بوجه عامهدار

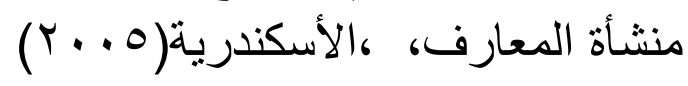

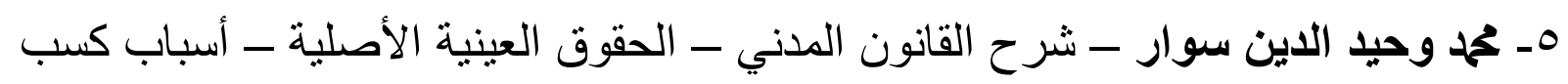

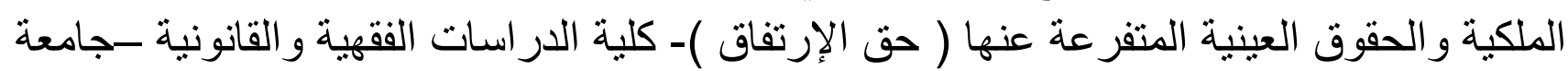

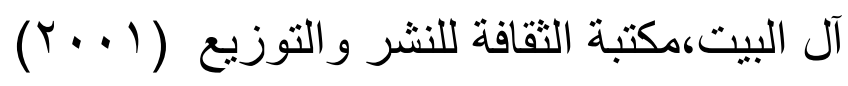

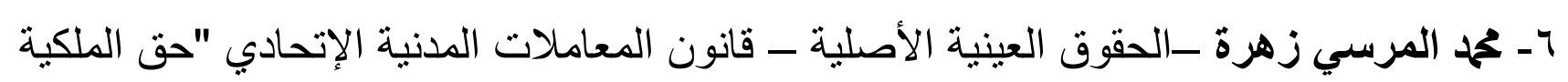

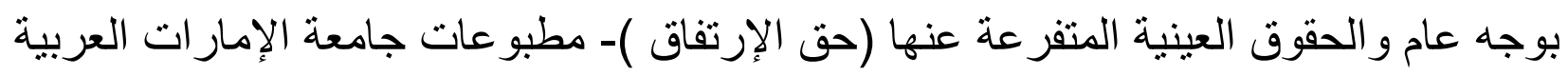
المتحدة - الطبعة الأولي ( 1999 (1)

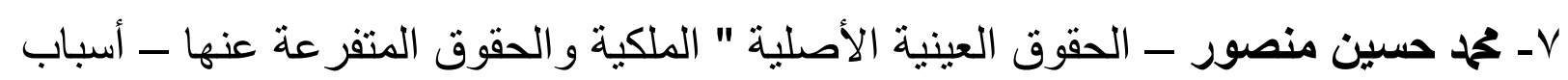

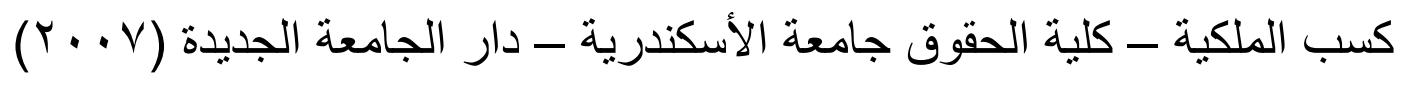




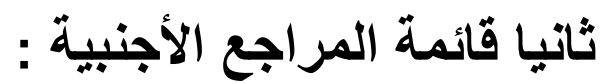

1-: James M. Pedowitz: SOLAR ENERGY EASEMENTS, Real Property, Probate and Trust Journal, Vol. 15, No. 4,( 1980)

2-: Endre Rex:Some Financial And Legal Aspects Of Solar Energy Usage:http://pledgingforchange.com/green/some-financial-and-legal-aspects-of-solarenergy-usage.php( the last visi 15/7/2019

3-K.K DuViver "SOLAR SKYSpace B,UniferSity of Denver Sturm, Collage of Law,(2014)

4- Adrian J. Bradbrook: "Australian and American Perspectives on the Protection of Solar and Wind Access" Natural Resources Journal,VOL: 28,(1988)

\section{5- Donald R. Richards Knud E. Hermansen:" MAINE ROADS AND}

EASEMENTS" Maine Law Review journal, Volume: 48 , Number: 2 Article: 3 ,(2018)

6- Gohn.wiliam.Gergacz:Solar Energy law : easements of access to Sunlight,(1980)

7- RULE, Troy "Airspace in a Green Economy" Law Review Journal,Vol:59, school of law ,University of Missouri ,(2011)

8- Reize ,G.L."A solar rights zoning guarantee seeking new law in old ,law ReviewJOURNAL N:3, Washingoton University (1976) concepts" 